\title{
A DEM study of powder spreading in additive layer manufacturing
}

\author{
Yahia M. Fouda ${ }^{1,2} \cdot$ Andrew E. Bayly $^{1}$ \\ Received: 22 February 2019 / Published online: 15 November 2019 \\ (c) The Author(s) 2019
}

\begin{abstract}
In this paper, discrete element method simulations were used to study the spreading of an idealised, blade based, powder coating system representative of the spreading of spherical, mono-sized, non-cohesive titanium alloy (Ti6AlV4) particles in additive layer manufacturing applications. A vertical spreader blade was used to accelerate a powder heap across a horizontal surface, with a thin gap between the blade and the surface, resulting in the deposition of a thin powder layer. The results showed that it is inevitable to deposit a powder layer with a lower packing fraction than the initial powder heap due to three mechanisms: shear-induced dilation during the initiation of powder motion by the spreader; dilation and rearrangement due to powder motion through the gap; and the inertia of the particles in the deposited powder layer. It was shown that the process conditions control the contribution of these three mechanisms, and that the velocity profile in the shear layer in front of the gap is critical to the final deposited layer packing fraction. The higher the mean normalised velocity in the shear layer the lower the deposited layer packing fraction. The gap thickness and the spreader blade velocity affect the properties of the deposited layer; with the former increasing its packing fraction and the latter decreasing it. The analysis presented in this study could be adapted to powders of different materials, morphologies and surface properties.
\end{abstract}

Keywords Additive manufacturing (AM) $\cdot$ Powder spreading $\cdot$ Discrete element method (DEM) $\cdot$ Dilation $\cdot$ Metal powders · Powder bed fusion

$\begin{array}{ll}\text { List of } & \text { symbols } \\ d & \text { Particle diameter } \\ t & \text { Time } \\ v & \text { velocity } \\ x, y, z & \text { Cartesian coordinates } \\ \delta & \text { Gap thickness } \\ \Delta \eta & \text { Change in packing fraction } \\ \eta & \text { Packing fraction }\end{array}$

\section{Subscripts}

1,2, 3 First, second and third packing fraction reduction mechanisms, respectively

back Interrogation region at the back of the spreader front Interrogation region in front of the spreader

Yahia M. Fouda

Yahia_Fouda@mans.edu.eg

Andrew E. Bayly

A.E.Bayly@leeds.ac.uk

1 School of Chemical and Process Engineering, University of Leeds, Leeds LS2 9JT, UK

2 Department of Mechanical Power Engineering, Faculty of Engineering, Mansoura University, Mansoura 35516, Egypt

$\begin{array}{ll}p & \text { Particle } \\ w & \text { Spreader } \\ \infty & \text { Final }\end{array}$

\section{Introduction}

According to the American Society for Testing and Materials (ASTM), additive manufacturing (AM) is defined as the process of joining materials to make objects from three-dimensional model data [1], usually in a layer upon layer fashion. Conventional subtractive manufacturing, on the other hand, removes material from a continuous billet. Additive manufacturing, often referred to as 3D printing, has been called a new industrial revolution [2] due to its intrinsic digital approach in manufacturing three-dimensional objects. Complex shapes can be easily created enabling the production of topology optimised components, which reduces the overall weight and material used and facilitates the manufacturing of shape-customised objects.

As implied by its name, powder bed fusion additive manufacturing techniques, such as Electron Beam Melting (EBM) and Selective Laser Melting (SLM), use the raw material in the form of powder. Powder bed fusion is 
capable of manufacturing metallic components; which are used in aerospace, medical and dental applications [3-6]. For both EBM and SLM, the manufacturing process starts by spreading or depositing a thin powder layer over a flat horizontal substrate inside an enclosed chamber. In this deposited powder layer, specific regions conforming to the geometry of the final manufactured object are melted using energy from either a laser or an electron beam. The melted powder solidifies when it cools down resulting in a continuous densified solid layer. Subsequently, the substrate and the powder cake attached to it are moved vertically downwards by an increment equal to the powder layer thickness in order to create a space for another layer. By sequentially repeating this process for a finite number of layers, a near-net shape product is manufactured.

It has been reported that the deposited powder layer properties, namely its uniformity and packing density, affect the final porosity and the bulk density of the fabricated part [4, $7,8]$. The input parameters of the spreading process, such as the powder characteristics and the machine operating conditions, can indeed affect the layer uniformity and density [4, 7-9]. Unfortunately, no rigorous theory describing the physics of powder spreading in AM has been reported in the literature. Previous studies investigated the effects of powder and process parameters on the whole additive manufacturing process [10-12]; an object is manufactured using powders with different characteristics and the final properties of this manufactured object are tested. This approach, despite being useful in developing empirical correlations describing the parameters of the manufacturing process, is not capable of analysing the complex multi-physics of the AM processes.

Numerical simulations can shed some light on the unknown physics of different AM processes. The flexibility of numerical simulations can help in reducing the number of experimental tests and obtain better physical understanding of emerging engineering processes. The Discrete Element Method (DEM) has proven itself as an excellent numerical tool for studying powder flow dynamics, and powder spreading in $\mathrm{AM}$ is no exception. DEM tracks the motion of each particle throughout the spatial and temporal computational domain, by solving its Newton's equations of motion. In order to calculate the interactions between the particles and between the particles and geometry, DEM resolves every particle-particle and particle-wall contact event. Thus, a typical DEM simulation generates data describing the dependent variables of individual particles; namely spatial positions, linear and angular velocities, and applied forces and torques; as functions of time. This allows access to quantitative results which might be difficult, expensive and time consuming to obtain from experiments.

DEM has been recently used to study the powder spreading dynamics in AM [13-16]. Parteli and Pöschel [13] performed DEM simulations of powder spreading using a cylindrical-shape spreader. The powder used consisted of non-spherical particles. Their results showed that increasing the spreading speed leads to an increase in the surface roughness of the deposited powder layer. They also found that powders with broader size distributions lead to larger values of layer surface roughness. Haeri et al. [14] used DEM simulations to study the spreading of rod-shaped polymer particles using a cylindrical roller spreader as well as a flat vertical blade spreader. They found, in agreement with Parteli and Pöschel [13], that the layer quality, quantified by the layer surface roughness and the packing density, deteriorates by increasing the roller or blade spreader velocity. They also found that the roller gives better layer quality compared to the spreader. Xiang et al. [15] used DEM simulations to study the formation of a powder bed consisting of 4000 spheres. The powder bed formation process had three consecutive stages: packing, layering and compression. Their results showed that the bed packing fraction and coordination number increase by increasing the bed height (initial layer thickness) and the bed compression. Haeri [16] used DEM simulations to optimise the geometry of the spreader in order to produce a powder layer with maximum packing fraction. These studies, despite being novel in investigating the spreading process, require further insights to understand the main mechanisms of spreading. The main aim of this paper is thus to develop, using DEM simulations, a better understanding of the dynamics of metal powder spreading in powder bed fusion additive manufacturing. A key objective is to understand the physical mechanisms affecting the quality of the deposited layer.

\section{Methodology}

\subsection{DEM}

As DEM is becoming a standard simulation tool of particulate processes, its fundamental details are not presented here and the reader is referred to the published literature [17, 18]. In this paper, the simulations were carried out using the commercial DEM software EDEM $^{\circledR}$; supplied by DEM Solutions, Ltd. The 'Hertz-Mindlin (no slip)' contact model of the EDEM software [19] was used to compute the particle-particle and particle-walls interactions.

\subsection{Materials}

In order to accurately simulate particle motion using DEM; the material properties of powder particles and enclosing walls are required as input parameters. Some of these parameters are, however, difficult to measure [20] and thus, they are often estimated [21-25]. A common approach used in DEM simulations is to calibrate the model predictions 
[26-31] with the static angle of repose, defined as the angle of inclination of the free surface to the horizontal of a conical bulk solid pile [31]. The DEM input parameters used in this study, see Table 1, predicted a static angle of repose of $18^{\circ}$; whereas the value of EBM titanium alloy (Ti6Al4V) powder, supplied by ARCAM, was measured by the authors at $20^{\circ}$. This confirms that the values presented in Table 1 are within a realistic range. Spherical mono-sized non-cohesive particles of $50 \mu \mathrm{m}$ diameter were used in all DEM simulations of this study. Ti6Al4V is a widely used material in metal AM and the powder is close to spherical as confirmed by SEM images of Ti6Al4V alloy particles used in ARCAM EBM machine [8]. Using mono-sized particles is a simplification of the real powder, though the median diameter of the number-based size distribution $(50 \mu \mathrm{m})$ is the same. This simplification allowed the layer thickness to be described using a single parameter, the number of particles inside it, which facilitate the comparison between different spatial regions in a single simulation as well as between different simulations. The analysis presented in this paper can thus be applied in the future to assess the exact powder size distributions encountered in AM applications.

Because the used DEM model did not consider cohesive forces, it is a valid assumption to reduce the shear modulus of both particles and walls in order to reduce the computational time [13]. The shear modulus was varied over three orders of magnitude (1,10 and $100 \mathrm{MPa})$ and the differences in the corresponding DEM predictions of powder kinematics were insignificant. Thus, a fixed value for the shear modulus of $1 \mathrm{MPa}$ was used, which resulted in a computational time step of $1.12 \mu \mathrm{s}$. These values resulted in a maximum particle contact-overlap of $0.2 \%$ of particle diameter for the maximum impact velocity of $0.1 \mathrm{~m} / \mathrm{s}$; which is within the recommended overlap range of accurate predictions, $0.1 \%$ to $0.5 \%$ $[32,33]$. A combination of sliding and rolling friction, as shown in Table 1, were used to partially supress the particle

Table 1 Materials properties and DEM input parameters

\begin{tabular}{ll}
\hline Particle size $(\mu \mathrm{m})$ & 50 \\
Particle material density $\left(\mathrm{kg} / \mathrm{m}^{3}\right)$ & 4300 \\
Walls material density $\left(\mathrm{kg} / \mathrm{m}^{3}\right)$ & 8500 \\
Particle shear modulus $(\mathrm{MPa})$ & 1 \\
Particle Poisson ratio & 0.3 \\
Wall shear modulus $(\mathrm{MPa})$ & 1 \\
Walls Poisson ratio & 0.3 \\
Particle-particle coefficient of restitution & 0.5 \\
Particle-wall coefficient of restitution & 0.5 \\
Particle-particle coefficient of sliding friction & 0.5 \\
Particle-wall coefficient of sliding friction & 0.5 \\
Particle-particle coefficient of rolling friction & 0.01 \\
Particle-wall coefficient of rolling friction & 0.1 \\
\hline
\end{tabular}

slip at the walls. The particle-wall rolling friction was one order of magnitude higher than that of the particle-particle to account for the relatively larger surface asperities of the walls. The density of the walls equals to that of stainlesssteel, a common material used to construct the internal walls of metal AM machines.

\subsection{Geometry}

A model geometry was used to represent a generic powder spreading system with a blade spreader, which is applicable to both EBM and SLM, see Fig. 1. The system consists of a vertical powder spreader blade (1), powder heap (2) and a horizontal build surface (3). The initial powder heap (2) is located over the build surface to allow the spreader to sweep the powder across the whole length of the build surface. A key feature of this spreading system geometry is the vertical gap between the spreader blade (1) and the build surface (3). This gap controls the thickness of the deposited powder layer, which corresponds to the resolution of the 3D printed component. Throughout the simulations performed in this paper, this gap was varied between 100 and $300 \mu \mathrm{m}$; which covers most of the layer thickness range of a typical electron beam melting (EBM) machine, between 50 and $200 \mu \mathrm{m}$ $[4,7]$ and is anticipated to be typical of the gap between the blade and the melted surface being recoated (this itself is determined by packing fraction of the deposited layer). Using the particle size and gap thickness of EBM systems does not deem the current study irrelevant to SLM systems, whose layer thickness is between 20 and $100 \mu \mathrm{m}$ [4] and particle size distribution between 15 and $45 \mu \mathrm{m}$ [4], because this layer thickness correlates with the particle diameter used and can be scaled down with it.

In the operation of a typical 3D printer, one aims to fill this gap with as much powder as possible; so as to minimise the voids in the $3 \mathrm{D}$ printed component and consequently avoid the deterioration of its mechanical properties [4]. However, a thick powder layer should be avoided because it reduces the resolution of the $3 \mathrm{D}$ printed component. If spherical particles with a specific size distribution are used, an intuitive requirement of their maximum diameter will be the gap thickness [34], because particles larger than the gap cannot get deposited on the build surface.

Due to the overwhelming computational requirements associated with simulating the process of powder spreading in a whole AM machine, whose dimensions are at least of the order of tens millimetres and contains billions of particles, the simulation geometry in this study was chosen to represent a longitudinal slice of a typical AM machine. The simulation employs periodic boundaries for the horizontal $y$-direction, which implies that the end effects in the $y$-direction are negligible. The simulation domain was further 


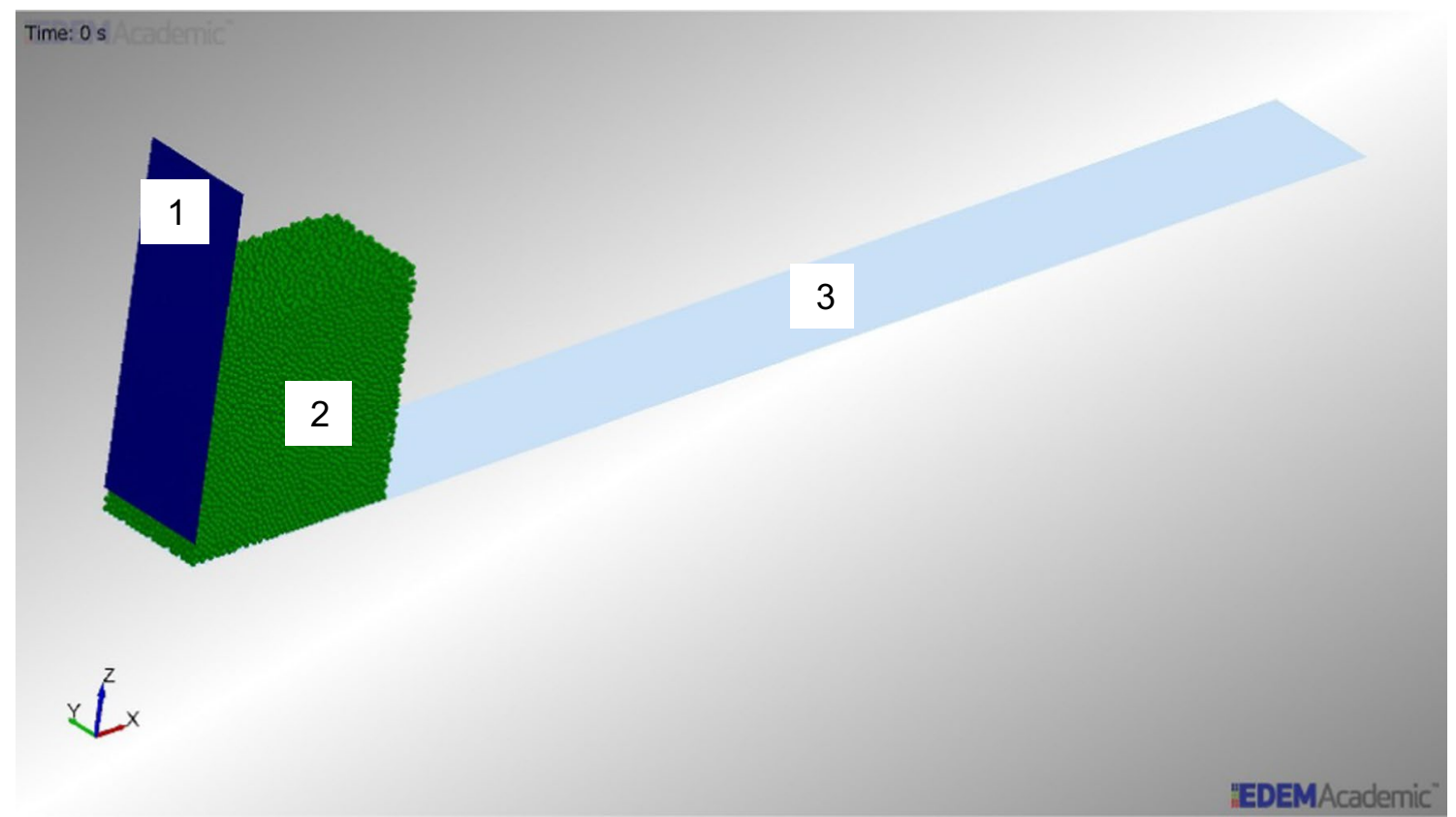

Fig. 1 Computational domain used in the simulations. (1) Spreader: $\Delta y=1 \mathrm{~mm}$, and $\Delta z=3 \mathrm{~mm}$. (2) Powder heap: $\Delta x=2 \mathrm{~mm}, \Delta y=1 \mathrm{~mm}$, and $\Delta z=2 \mathrm{~mm}$. (3) Build surface: $\Delta x=12 \mathrm{~mm}$ (2 mm underneath the

reduced by considering a $12 \mathrm{~mm}$ length in the horizontal $x$-direction, with $10 \mathrm{~mm}$ effective length monitored for the analysis of layer deposition.

\subsection{Initial conditions}

The initial powder heap was generated at the beginning of the simulation using the raining method [16]. Particles were generated from a horizontal plane and left to fall downwards under the effect of gravity in a bounded box opened from the top. Subsequently, the enclosing side walls were removed, resulting in an unconfined powder heap which is supported from below by the build surface and from the left by the spreader (Fig. 1). The total number of particles generated was 37,832 with an average packing fraction of $60.3 \%$, slightly below the random closed packing $(64 \%)$ and wellbelow the hexagonal crystalline packing (74\%).

The powder spreading simulation was initiated by instantaneously moving the spreader in the positive $x$-direction with a constant specified velocity (Fig. 1). As the spreader moves with a constant velocity from the left to the right across the domain, it pushes the powder heap in front of it. The powder is consequently deposited over the build platform throughout the longitudinal length in the $x-y$ plane. The powder heap has a cuboid shape initially, because the heap was created using a confined box (Fig. 1). In real powders, the heap would have a triangular shape because the particles will slip and roll over each other until the heap powder heap and $10 \mathrm{~mm}$ monitored for spreading), and $\Delta y=1 \mathrm{~mm}$. The offset between the bottom of the spreader blade (1) and the build platform (2) is equal to the gap thickness $(\delta)$

reaches the static angle of repose. However, the simulations performed after the heap had settled did not show any significant differences in dynamics or predicted later packing fraction. Thus, all simulations were performed with the cuboid heap in order to reduce the computational time; by eliminating the step of powder heap avalanching.

\section{Results and discussion}

\subsection{Mechanics of powder spreading}

In this section, a benchmark simulation using a gap thickness of 4 particle diameters $(\delta=200 \mu \mathrm{m})$ and a spreader velocity of $100 \mathrm{~mm} / \mathrm{s}$ is presented. The effects of varying the gap thickness and spreader velocity are presented in Sect. 3.2.

Figure 2 shows snapshots of powder motion in the spreading process at different time frames. The simulation starts with the powder heap positioned at the left end of the domain in front of the spreader (Fig. 2a). Figure $2 b$ shows that the heap avalanches and assumes a triangular prism shape during the motion of the spreader. The moving particles inside the powder heap are supported from the left by the powder spreader, which moves with a constant velocity from the start of the simulation to its end. It is clear from time $0.01 \mathrm{~s}$ onwards that as the powder heap moves with the spreader down the build surface, particles inside the gap between the bottom of the spreader and the build surface get detached 
Fig. 2 Snapshots of powder motion in the spreading process at different time frames. a $0.0 \mathrm{~s}$. b 0.01 s. c 0.02 s. d 0.03 s. e 0.09 s. f 0.122 s. $v_{w}=100 \mathrm{~mm} / \mathrm{s}$, $\delta=4 d$

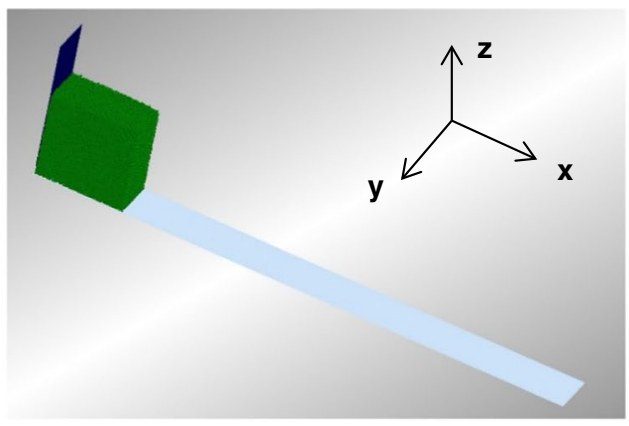

(a)

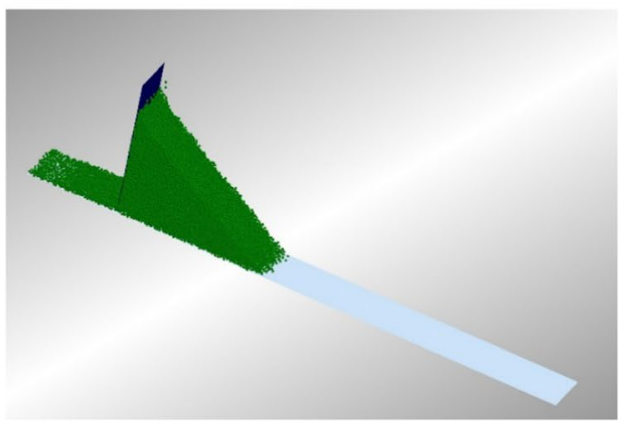

(c)

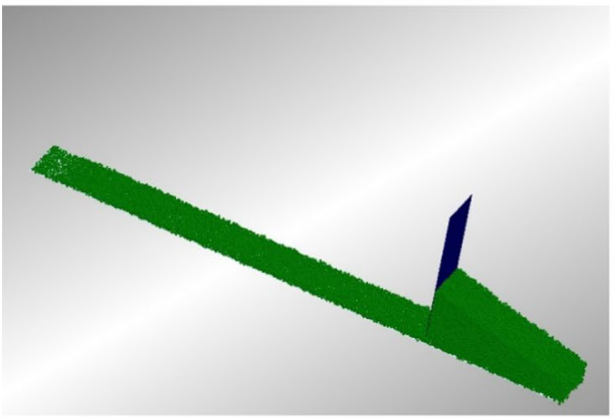

(e)

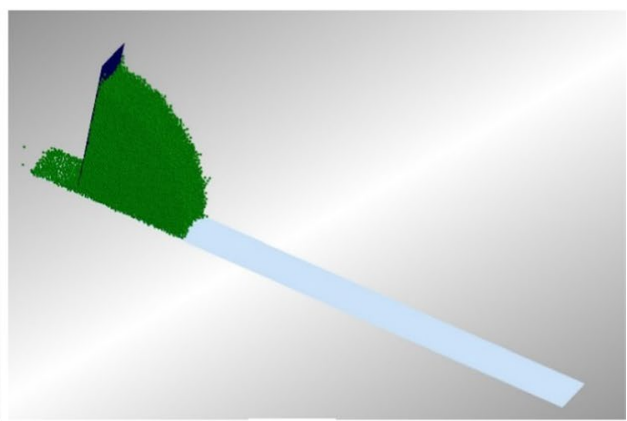

(b)

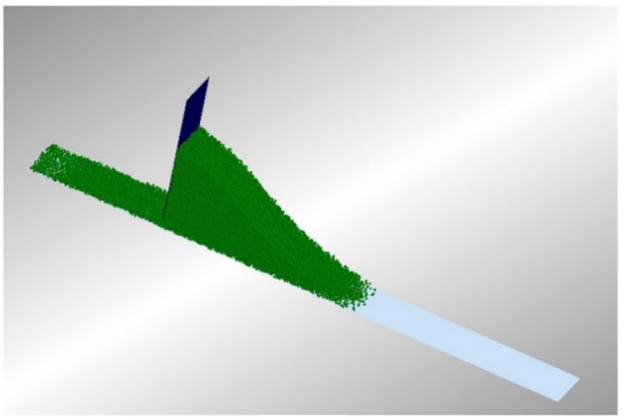

(d)

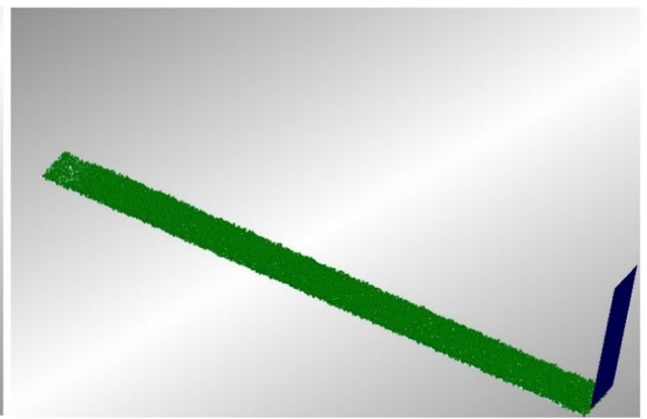

(f) from the powder heap and deposited over the build surface. At time $0.09 \mathrm{~s}$, the powder heap starts leaving the right end of the domain and the powder has covered the whole build surface. At the end of the simulation at time $0.122 \mathrm{~s}$, the spreader and the powder heap have left the domain and the powder layer has covered the whole build surface.

Figure 3 shows the effect of the horizontal position of the build surface on the final layer packing fraction, $\eta_{\infty}$, which is calculated, based on the gap thickness $(\delta)$ i.e. number of particles in the region of interest multiplied by the volume of a single particle, divided by the gap volume of this region. The variation in the packing fraction is small with a relative standard deviation of $4 \%$, implying that the spreading process results in a uniform powder layer throughout the longitudinal direction of the build surface.

Figure 4 shows the normalised number distribution of the deposited particles with the layer height. There is a peak at $d / 2$ representing the first layer of particles that are touching the build surface, and a broader peak at $\left(\frac{1}{2}+\frac{\sqrt{2}}{\sqrt{3}}\right) \boldsymbol{d}$, which indicates that there is some close packed periodicity in the system which might be anticipated (the layer spacing in a close packed system is equal to $\left.\frac{\sqrt{2}}{\sqrt{3}} d\right)$. The third peak is far more dispersed and lower than $\left(\frac{1}{2}+\frac{2 \sqrt{2}}{\sqrt{3}}\right) \boldsymbol{d}$ which would be seen for the third layer of a close packed system. The region above $~ 2.2$ particle diameters does not have any particles in it. This leads to a final packing fraction of $26 \%$ based on the gap thickness. To get a feel for the density of the packing it is useful to compare the particle distribution to a close packed system. A single close packed layer would give a packing fraction of $15.1 \%$ in this system with a gap height of $4 d$, thus $26 \%$ is equivalent to $26 / 15.1=1.72$ close packed layers. An effective porosity of the first layer versus a close packed system can be also be calculated, it contributes 
(a)

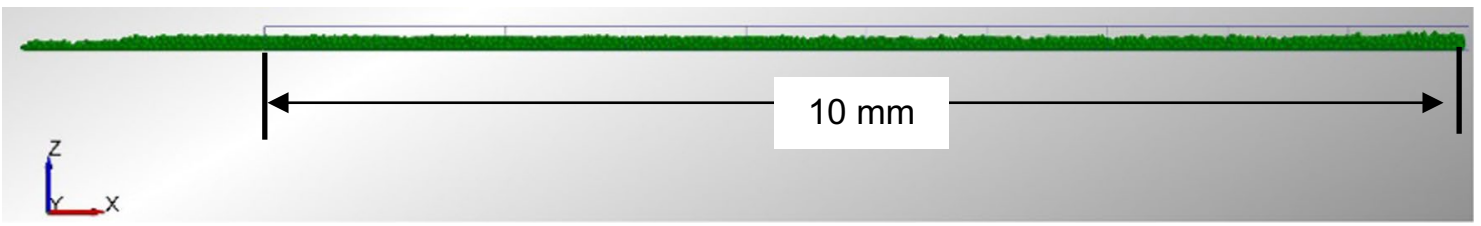

(b)

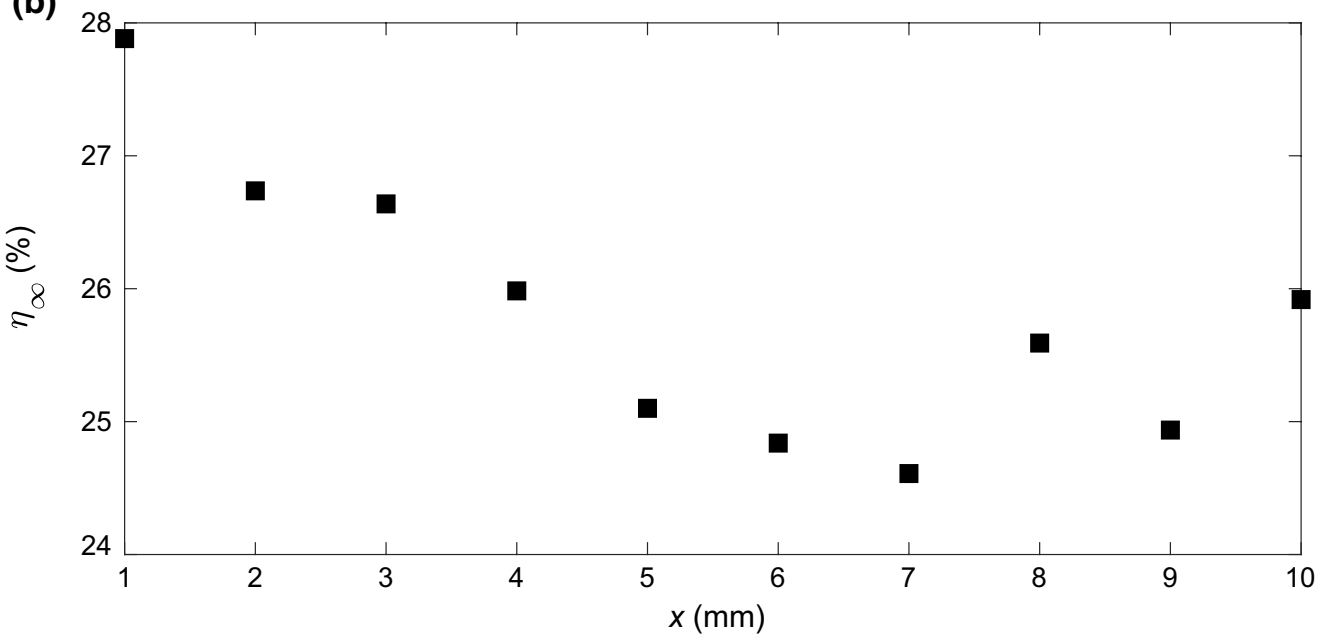

Fig. 3 Effect of the horizontal position of the build surface on the final layer packing fraction. Every data point is calculated over a $1 \mathrm{~mm}(20 d)$ bin of the $x$-direction and the whole gap thickness $(\delta=4 d)$ in $z$-direction, $v_{w}=100 \mathrm{~mm} / \mathrm{s}$

Fig. 4 Normalised number distribution of the deposited particles with the layer height. Every data point is calculated over a $0.005 \mathrm{~mm}(0.1 d)$ bin in the $z$-direction and the last $10 \mathrm{~mm}$ of spread length in the $x$-direction. $v_{w}=100 \mathrm{~mm} / \mathrm{s}$, $\delta=4 d$. Theoretical heights of close packed layers indicated

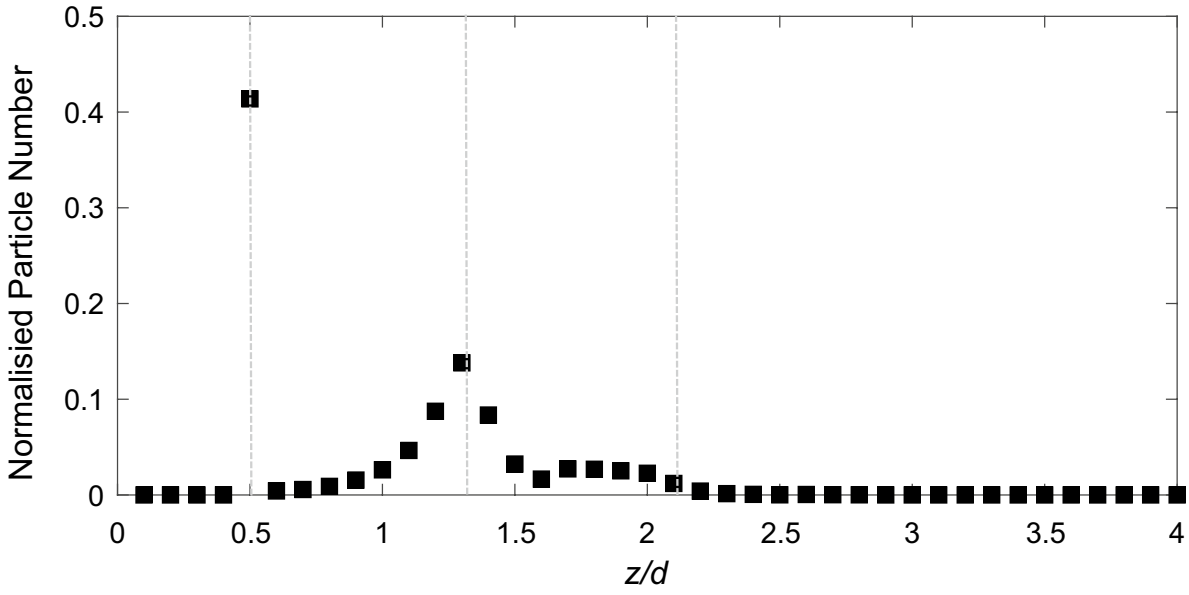

$10.7 \%$ to the final packing fraction and contains $71 \%$ of the particles that a close packed layer would.

The final deposit packing fraction of $26 \%$ is well below the $60 \%$ packing fraction of the initial powder heap. Consequently, it is worth studying the time dependent dynamic behaviour of the spreading process to understand the causes for significant reduction of the packing fraction, and height, of the deposited powder layer compared to that of the initial powder heap.
Figure 5 shows the packing fraction of two different moving interrogation regions in the vicinity of the spreader and the build surface with time. These two regions were chosen to move with the same velocity as the spreader so that their dynamic behaviour relative to the spreader can be analysed. The first region is in front of the spreader and the second region is at its back. Both regions have the same volume: five particle diameters in the longitudinal $x$-direction and the height equal to the gap thickness (four particle 


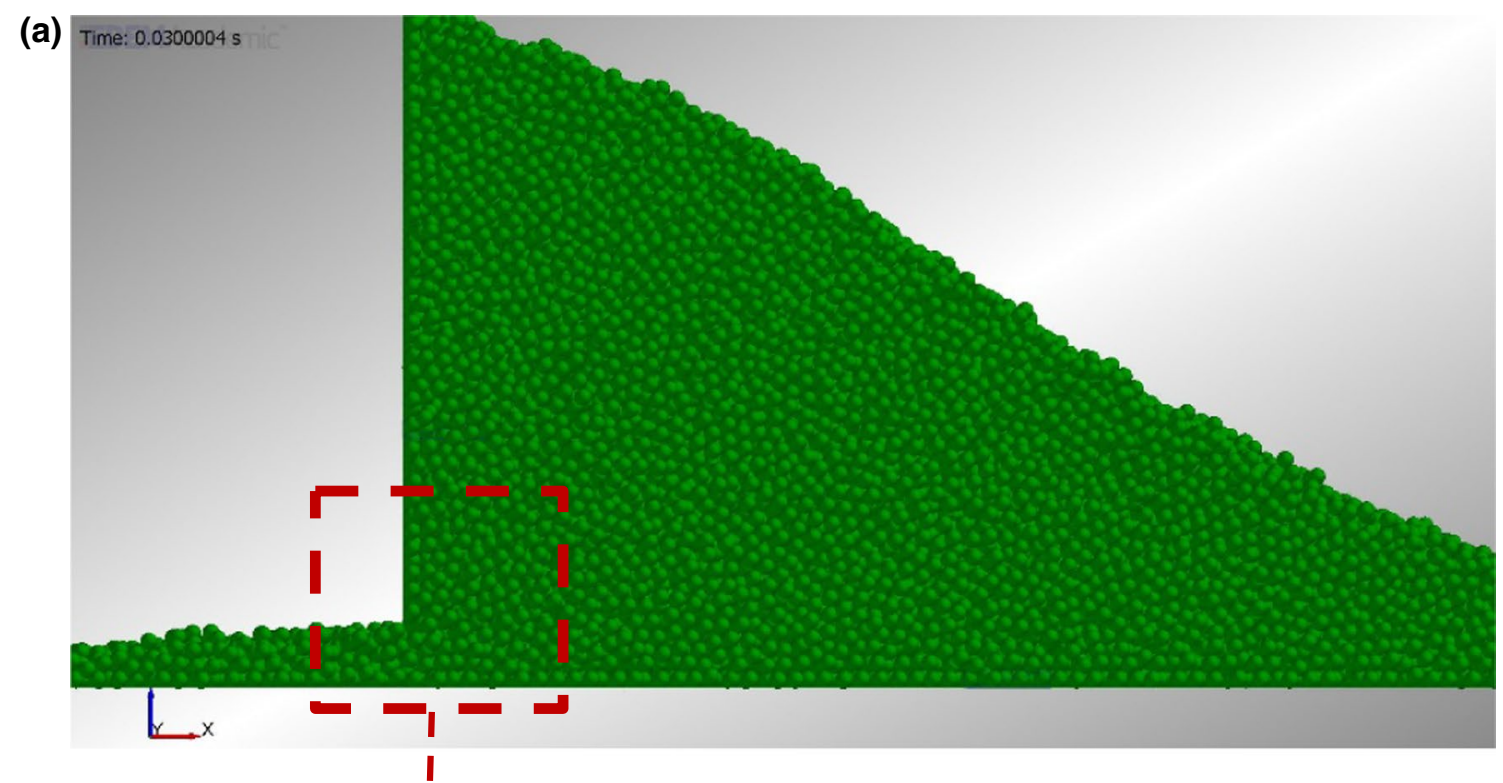

(b)
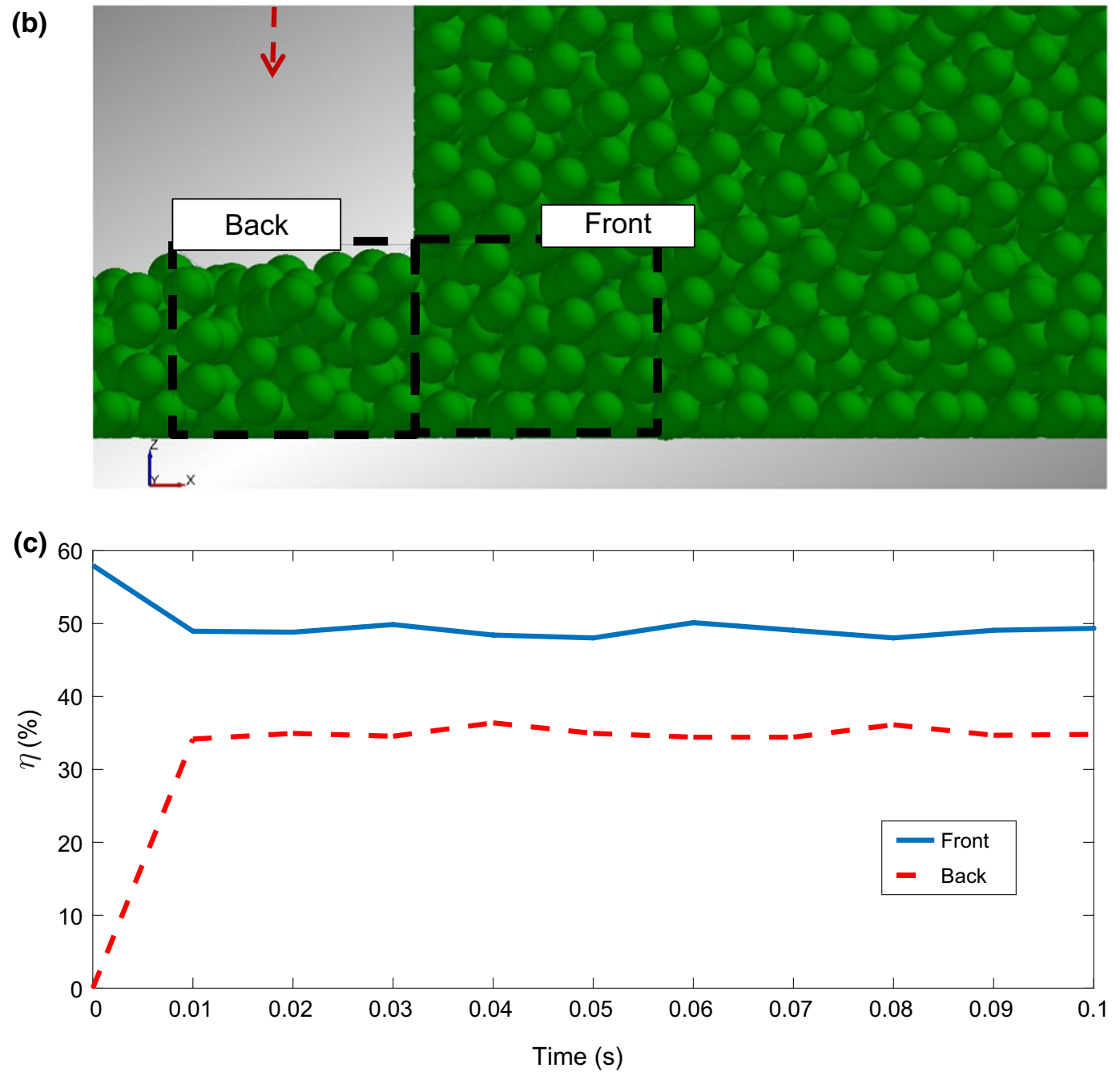

Fig. 5 Packing fraction of two different moving interrogation regions in the vicinity of the spreader and the build surface with time, $v_{w}=100 \mathrm{~mm} / \mathrm{s}, \delta=4 d$ 
diameters), see Fig. 5b. In Fig. 5c, two instances in which the packing fraction decreases are observed. The first instance is observed in front of the spreader; during the transient between 0.0 and $0.01 \mathrm{~s}$ the packing fraction drops from 58 to $49 \%$. The second instance is observed when the particles move through the gap to the back of the spreader; throughout the quasi-steady state between 0.01 and $0.1 \mathrm{~s}$, it is clear that the packing fraction in front of the spreader (49\%) is consistently higher than that at its back (34\%).

Figure 6 shows the average particle $x$-velocity in front of the spreader with time. In this analysis, the region in front of the spreader is divided into four sub-layers; each with a height of one particle diameter, see Fig. 6a. In Fig. 6b, the average particle $x$-velocity of each sub-layer experiences a transient period up to $0.01 \mathrm{~s}$, in which the particles are accelerated from rest, and then a quasi-steady state is reached. A shear velocity profile develops at $0.01 \mathrm{~s}$ with the $x$-velocity increasing with height above the build surface. The velocity of an undisturbed powder sub-layer, above the gap by a distance equal to five particle diameters $(0.25 \mathrm{~mm})$, is equal to the spreader velocity $(100 \mathrm{~mm} / \mathrm{s})$ in the quasisteady state. The shear forces, exerted on the particles in the region in front of the spreader, are due to the contact forces from the particles above and the friction resistance from the build surface below. This creates a vertical variation in the horizontal $x$-velocity of the sub-layers inside the gap. There are small temporal fluctuations in $x$-velocity within each sub-layer, during the quasi-steady behaviour, as the number of particles and contacts within the sub-layer change with time.

(a)

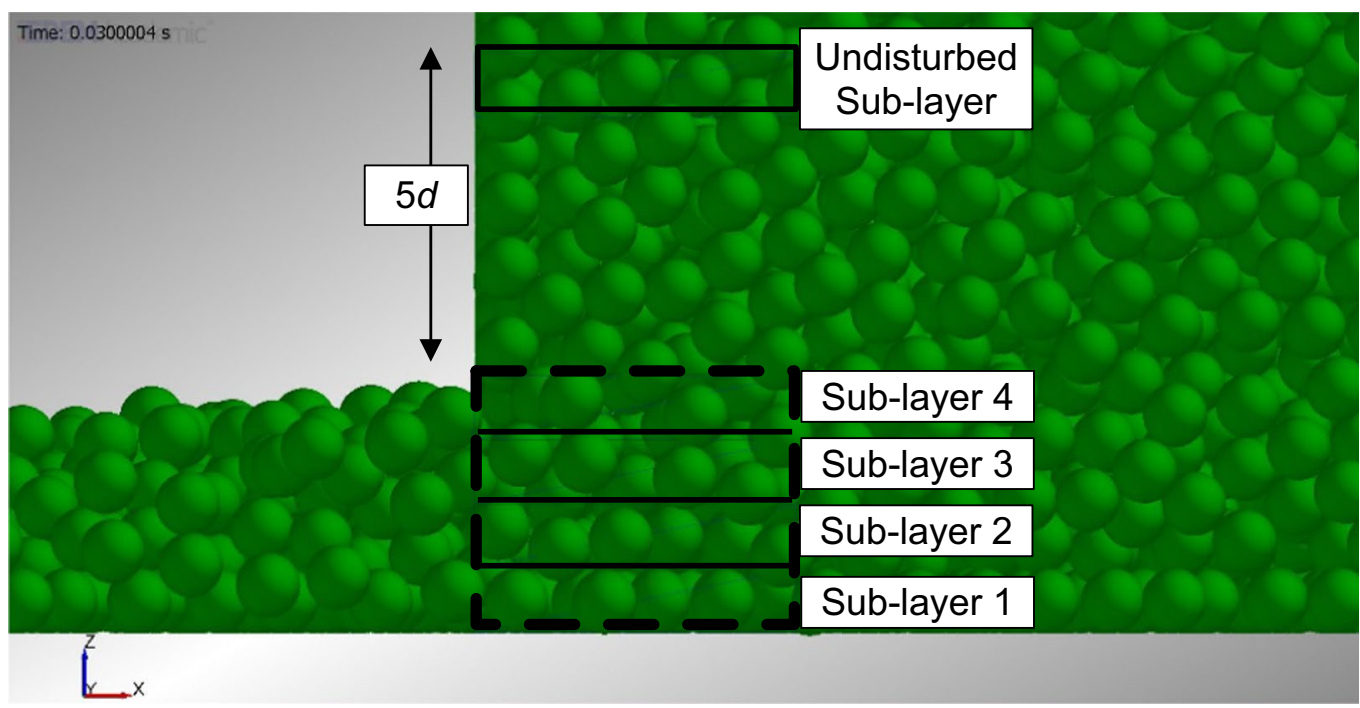

(b)

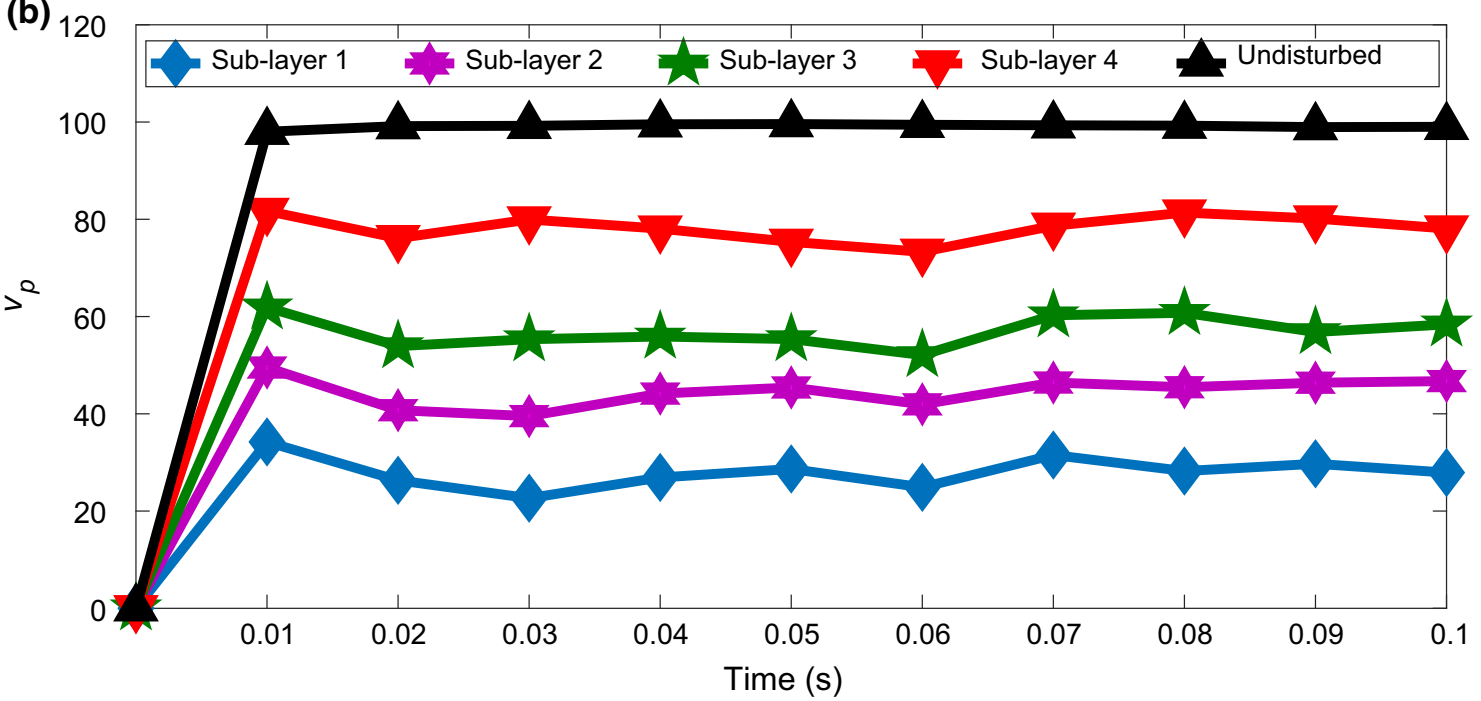

Fig. 6 Particle $x$-velocity in each sub-layer in front of the spreader with time, $v_{w}=100 \mathrm{~mm} / \mathrm{s}, \delta=4 d$ 
Figure 7 shows this data as a time averaged velocity profile. The normalised velocity varies between 0 and 1 across a thickness of 9 particle diameters, signalling the existence of a shear band, a narrow zone occurring in sheared-granular material where all the strain is localised. The thickness of the shear band is consistent with the literature of granular shear flows [35-38]. It is clear that the shear rate is higher across the gap compared to that above it; the velocity reaches $80 \%$ at the top sub-layer inside the gap (4 particle diameters) before it reaches $100 \%$ of the spreader velocity at 9 particle diameters up from the build surface.

When a granular assembly is sheared; it dilates, i.e. its packing fraction decreases. This effect was first noticed by Reynolds [39] and has been reported in the literature of sheared granular media [40-45] and flow in hoppers [46-51] or silos [52]. Shear-induced dilation occurs because when a granular medium is subjected to a local shear it must expand by creating more void spaces to allow the grains to pass by each other and overcome the grains interlocking. As confirmed by Fig. 6, powder spreading in AM involves shearing the powder in front of the spreader to initiate the motion and accelerate the powder from rest; thus, the powder should dilate, which explains the packing fraction reduction observed in front of the spreader during the transient time between $0.0 \mathrm{~s}$ and $0.01 \mathrm{~s}$ in Fig. 5. The packing fraction in front of the spreader is almost steady (Fig. 5) when the corresponding particle velocity is steady (Fig. 6) between 0.01 and $0.1 \mathrm{~s}$.

The reduction in the packing fraction at the back of the spreader compared to that at the front (Fig. 5) is due to the powder flow through the gap. If the system is viewed in a moving reference frame whose velocity is equal to that of the spreader, the particles $x$-velocity in sub-layers in front of the spreader will be towards the left. Thus, the motion of the particles from the region in front of the spreader to that at its back is reminiscent of granular flow through orifices. It is well known that in such situations the powder dilates as it exits the orifice [46, 47, 51, 53, 54], due to the increase in the shear rate and increase in velocity. For powder spreading in AM, the shear rate across the gap becomes higher than the average shear rate in front of the spreader, because the shear zone tapers from the height of the shear band (9 particle diameters) to the height of the gap (4 particle diameters). The particle velocity, in the reference frame of the blade, also begins to increase as the vertical confining forces are released and the base accelerates the particles away from the gap.

Figure 8 compares the quasi-steady average packing fraction in the horizontal sub-layers at the front and the back of the spreader. The data is also tabulated along with the velocity profile in Table 2 . For the first and the second sub-layers, the packing fraction is approximately the same at the front and the back of the spreader. However there is a clear reduction in packing fraction in the third and fourth sub-layer, as the particles are forced under the spreader. Similar transverse packing fraction profiles in which the powder dilation is more pronounced close to the edges of the orifice were reported in granular flow discharging from hoppers [46].

To understand the dynamics in this region and the reason for the localization of the packing fraction changes it is useful to look at the change in the velocity profile which is linked to changes in packing fraction.

To achieve mass conservation, then for any vertical plane at or behind the orifice

$\delta$

$\int_{o}^{\delta} \eta(z)\left(v_{w}-v_{p}(z)\right) \mathrm{d} z=\eta_{\infty} v_{w}$

where $\eta(z)$ and $v_{p}(z)$ are the time-averaged packing fraction and $x$-velocity at height $z$, and $v_{w}$ is the spreader velocity. $\eta_{\infty} v_{w}$ is a constant and represents the total mass flux leaving the gap, $\eta_{\infty}$ being the final layer packing fraction when the particle velocity is zero. This would also hold in front
Fig. 7 Effect of height on the normalised quasi-steady average $(0.01 \mathrm{~s}$ to $0.1 \mathrm{~s})$ particle $x$-velocity in front of the spreader. $v_{w}=100 \mathrm{~mm} / \mathrm{s}, \delta=4 d$. The data are binned at the top edges of the sub-layers

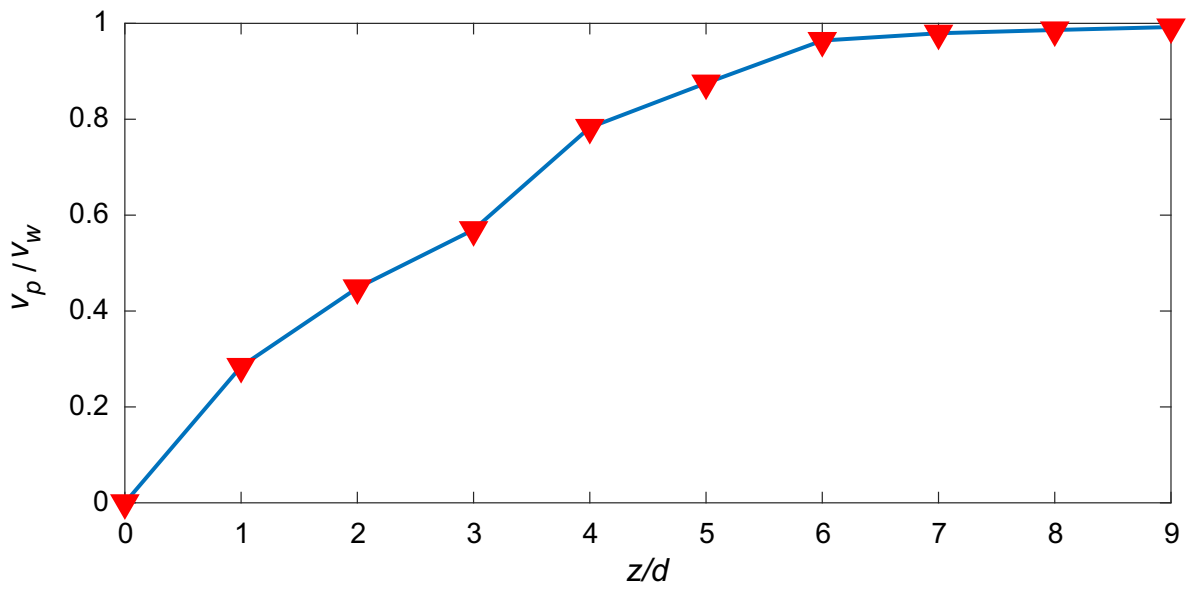




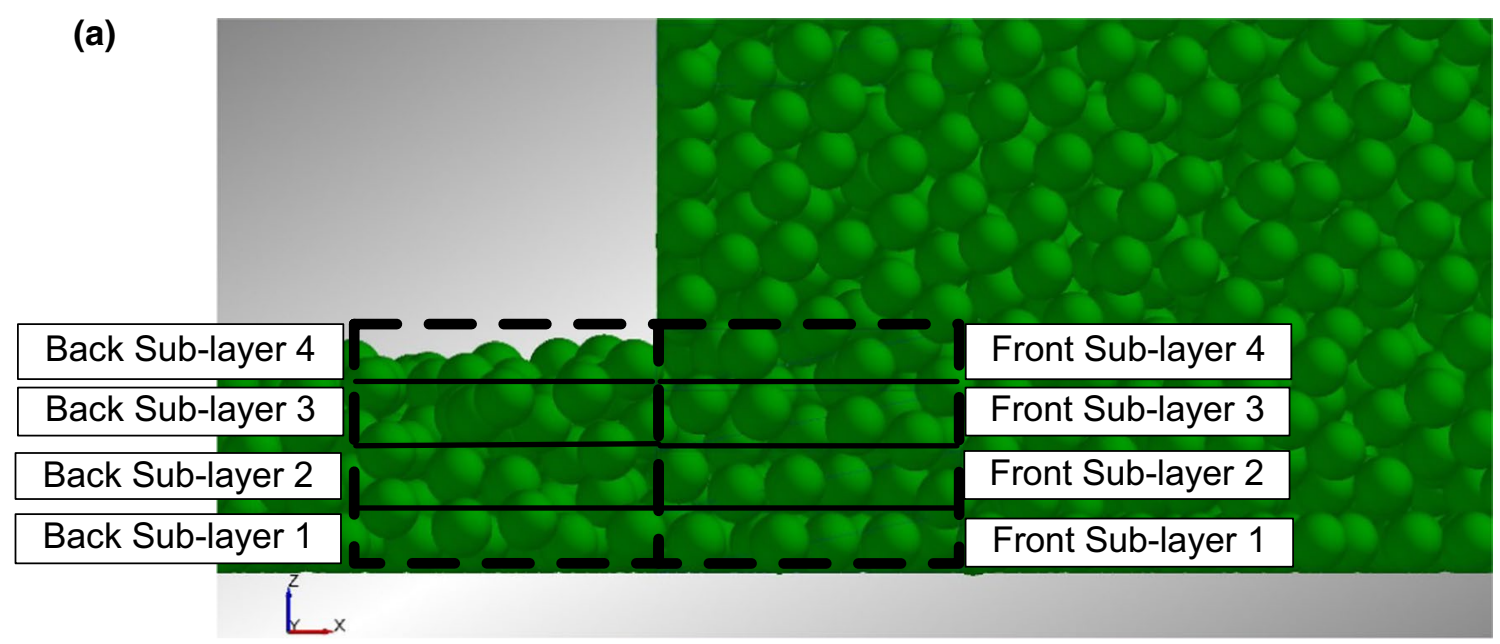

(b)

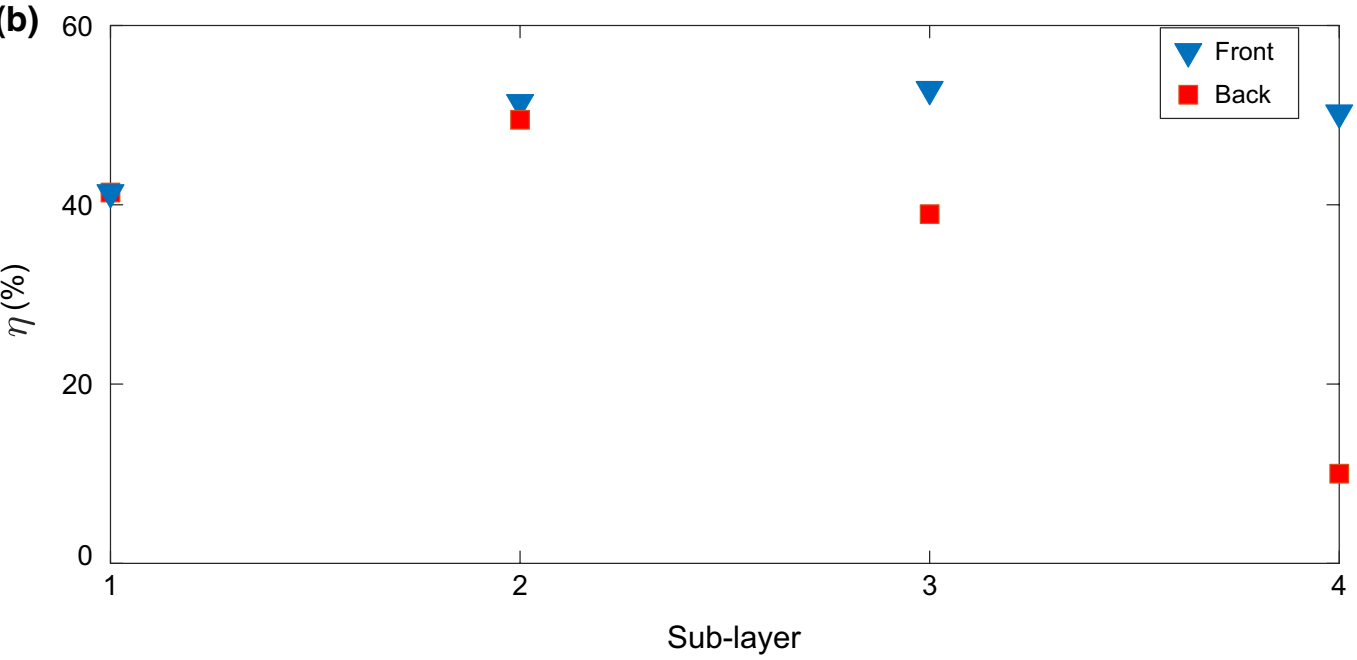

Fig. 8 Quasi-steady average $(0.01 \mathrm{~s}$ to $0.1 \mathrm{~s})$ packing fraction in different horizontal sub-layers at the front and the back of the spreader. $v_{w}=100 \mathrm{~mm} / \mathrm{s}, \delta=4 d$

Table 2 Mass flux analysis in sub-layers before and after spreader

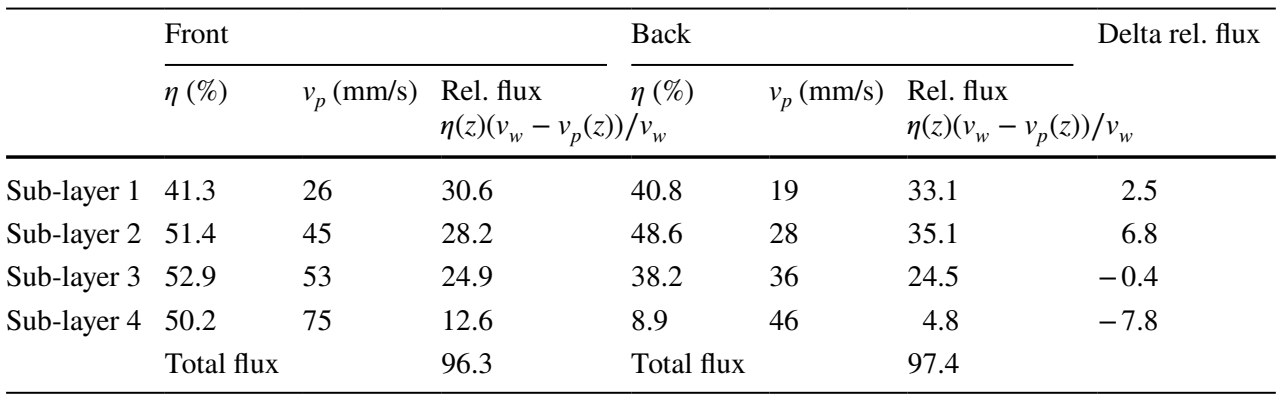

of the orifice if the vertical velocity is low compared to the horizontal velocity. As can be seen from Table 2, this is true indicating, the comparisons of the total fluxes indicating an only $\sim 1 \%$ increase in flux, which must be due to a vertical entry into the front zone from above.
Interestingly the packing fraction at the back of the blade in the lower layers does not decrease as we might expect due to the particle deceleration observed (particle acceleration in the ref. frame of the spreader). This is probably because the shear rate is similar, consequently the dynamic packing 
fraction remains roughly constant, as the 'holes' caused by the dilation due to the change in velocity are filled by particles from the upper layers falling into gaps in the lower layers. This filling of the lower layers also accounts for the big fall in the packing fraction of the top layer. A vena contractor type effect is also taking place, aided by the need for particles with centres between $3.5 d$ and $4.0 d$, in the front zone being forced down into the lower zones as they flow through the orifice and consequently the position of their centres must be below $3.5 d$. This is seen in the change in the relative sub-layer mass fluxes from the front to the back, these would remain equal if the vertical velocities were zero, however the differences show that there must be significant vertical flux as the particles pass from the front to the back. The relative flux in sub-layer 2 increases by around $20 \%$, whereas the relative flux in sub-layer 3 remains constant as it loses mass to sub-layer 2 and gains mass from sub-layer 4. Additionally it would also be anticipated that there be a higher dilation in the top layer due to the far larger change in velocity for these layers, this is reflected in the relative flux as a function of height, the top layer having a significantly reduced relative flux compared to the bottom layer.

The final packing fraction of the powder layer deposited over the build surface further decreases compared to that at the back of the spreader. The average packing fraction at the back of the spreader during the spreading process is $35 \%$ (Fig. 5), whereas the average packing fraction of the final deposited powder layer is $25.8 \%$ (Fig. 4). This is because, as shown in Table 2, the particles are still moving when they exit the gap. This is also shown by Fig. 9, which shows the average particle $x$-velocity in a fixed interrogation region of the build surface ( $3 \mathrm{~mm}$ to $4 \mathrm{~mm}$ along $x$-axis). At time of $0.03 \mathrm{~s}$, the spreader is at the edge of this interrogation region. At time of $0.04 \mathrm{~s}$, the spreader has moved across the interrogation region; the spreader is at $4 \mathrm{~mm}$ across the build surface. However, the curve shows that particles are moving even after the spreader has passed across the interrogation region; particles have a non-zero velocity for times higher than $0.04 \mathrm{~s}$. This indicates that the particles at the back of the spreader keep moving after they get detached from the main powder heap due to their inertia. Thus, the packing fraction inside the layer further decreases due to the relaxation time required to stop the moving particles via the interactions of other particles below them. The top sub-layer has the highest slip velocity compared to the others. The Figure shows that the velocities of the particles in all sub-layers collapse to zero at one point $(0.05 \mathrm{~s})$.

The results presented in this section have revealed three key mechanisms causing the reduction in the packing fraction of the deposited layer compared to the initial powder heap. The first mechanism is observed when the packing fraction of the powder in front of the spreader during the quasi-steady state is less than that of the initial power heap (Fig. 5). This mechanism is due to the shear-induced dilation during the initiation of powder motion by the spreader (Fig. 6). The second mechanism is observed when the packing fraction at the back of the spreader is less than that at the front of the spreader (Fig. 5). This is due to dilation when the powder moves through the gap (Fig. 8), which is reminiscent to powder flow through orifices. The third mechanism is observed when the packing fraction of the deposited powder layer is less than that of the powder at the back of the spreader. This mechanism is due to the inertia of the particles, and the necessary change in packing fraction required to conserve mass as the velocity changes; in the reference frame of the spreader they are being accelerated to the speed of the base as they are detached from the powder heap (Fig. 9). The system exhibits some similarities to simple shear and flow through orifices.

\subsection{Effect of process parameters}

For the given geometry and configuration shown in Fig. 1, the process of powder spreading in AM involves three sets of input parameters describing the powder, machine and process characteristics. Powder characteristics include particle
Fig. 9 Average particle $x$-velocity against time in sublayers at a fixed region in the $x$-coordinate ( 3 to $4 \mathrm{~mm}$ off the start of the build surface) on the build surface. $v_{w}=100 \mathrm{~mm} / \mathrm{s}$, $\delta=4 d$

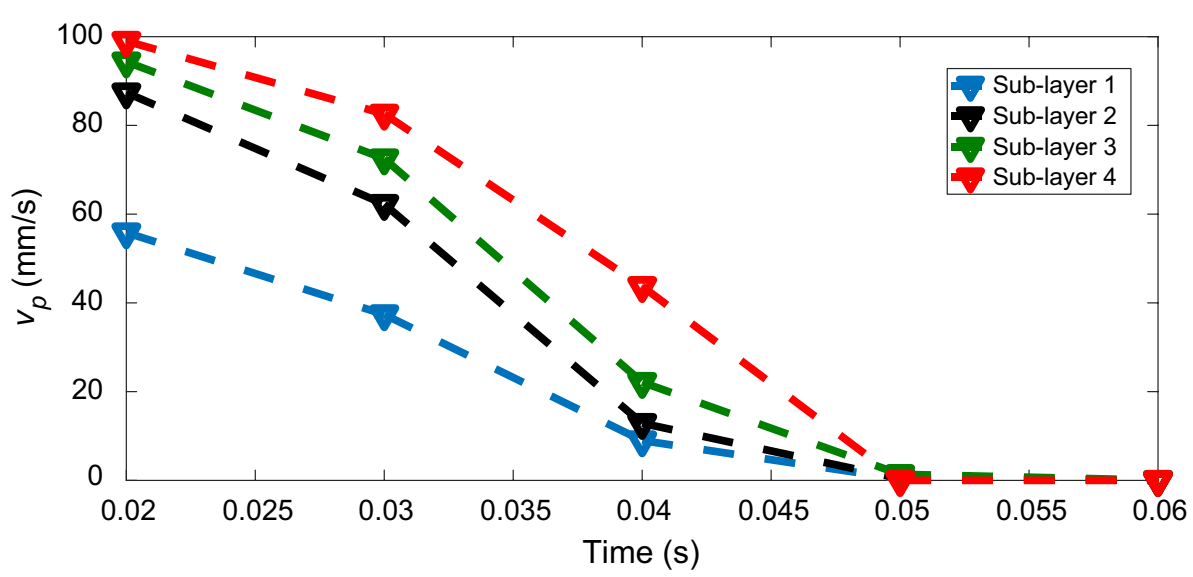


size distribution, volumetric shape, material and surface properties. The machine parameters include the geometry of spreader, and the material properties of both the spreader and the build surface. The process parameters include the gap thickness and the spreader velocity. It is rather beyond the limits of any single study to thoroughly investigate the effect of all these parameters. Thus, the focus here is on two key process parameters: the gap thickness and the velocity of the spreader; as a key objective of this study is to quantify the quality of the deposited layer, the three mechanisms causing the reduction of deposited layer packing fraction are monitored. The metrics used to quantify these mechanisms are defined as follows:

The first packing fraction reduction mechanism $\Delta \eta_{1}$, due to shear-induced dilation during the initiation of powder motion by the spreader, is defined as:

$\Delta \eta_{1}(t)=\left(1-\frac{\eta_{\text {front }}(t)}{\eta_{\text {front }}(0)}\right) \times 100$

where $\eta_{\text {front }}(0)$ is the initial packing fraction of the stagnant powder heap before spreading. It is calculated over the region in front of the spreader as shown in Fig. 5. $\eta_{\text {front }}(t)$ is the time-varying packing fraction in front of the spreader.

The second packing fraction reduction mechanism $\Delta \eta_{2}$, due to dilation when the powder moves through the gap, is defined as:

$\Delta \eta_{2}(t)=\left(\frac{\eta_{\text {front }}(t)-\eta_{\text {back }}(t)}{\eta_{\text {front }}(0)}\right) \times 100$

where $\eta_{\text {back }}(t)$ is the time-varying packing fraction of a moving interrogation region at the back of the spreader, see Fig. 5. In addition, the above equation is used to monitor the packing fraction in different sub-layers inside the gap as shown in Fig. 8.
The third packing fraction reduction mechanism $\Delta \eta_{3}(t)$, due to mass conservation as the horizontal velocity of the deposited layer changes, is defined as:

$\Delta \eta_{3}(t)=\left(\frac{\eta_{\text {back }}(t)-\eta_{\infty}}{\eta_{\text {front }}(0)}\right) \times 100$

where $\eta_{\text {final }}$ is the final packing fraction of the deposited layer.

The reduction in packing fraction for each mechanism is time-averaged over the quasi-steady state period as:

$\Delta \eta_{i}=\overline{\Delta \eta_{i}(t)}$

The overall reduction in the packing fraction $\Delta \eta$ is the summation of the three mechanisms as:

$\Delta \eta=\Delta \eta_{1}+\Delta \eta_{2}+\Delta \eta_{3}$

\subsubsection{Gap thickness}

Here, the gap thickness is varied from 2 to 6 particle diameters $(0.01-0.03 \mathrm{~mm})$, while keeping the velocity of the spreader constant at $100 \mathrm{~mm} / \mathrm{s}$. Figure 10 shows the effect of the gap thickness on the final layer packing fraction; the layer packing fraction increases almost linearly with the gap thickness. Figure 11 shows the effect of gap thickness on the three packing fraction reduction mechanisms. For the first mechanism, shear-induced dilation during the initiation of powder motion by the spreader, the reduction in packing fraction fluctuates between 12 and $20 \%$ for different gap thicknesses with no clear trend. For all gap thicknesses, the second mechanism, dilation and rearrangement when the powder moves through the gap, is the most significant one. However, its relative importance varies with the gap thickness. It reaches $40 \%$ for a gap thickness of 2 particle diameter, whereas it is $18 \%$ for a layer thickness of 6 particle diameter. The third mechanism, due to particle deceleration and mass conservation,
Fig. 10 Effect of gap thickness on the final layer packing fraction, $v_{w}=100 \mathrm{~mm} / \mathrm{s}$

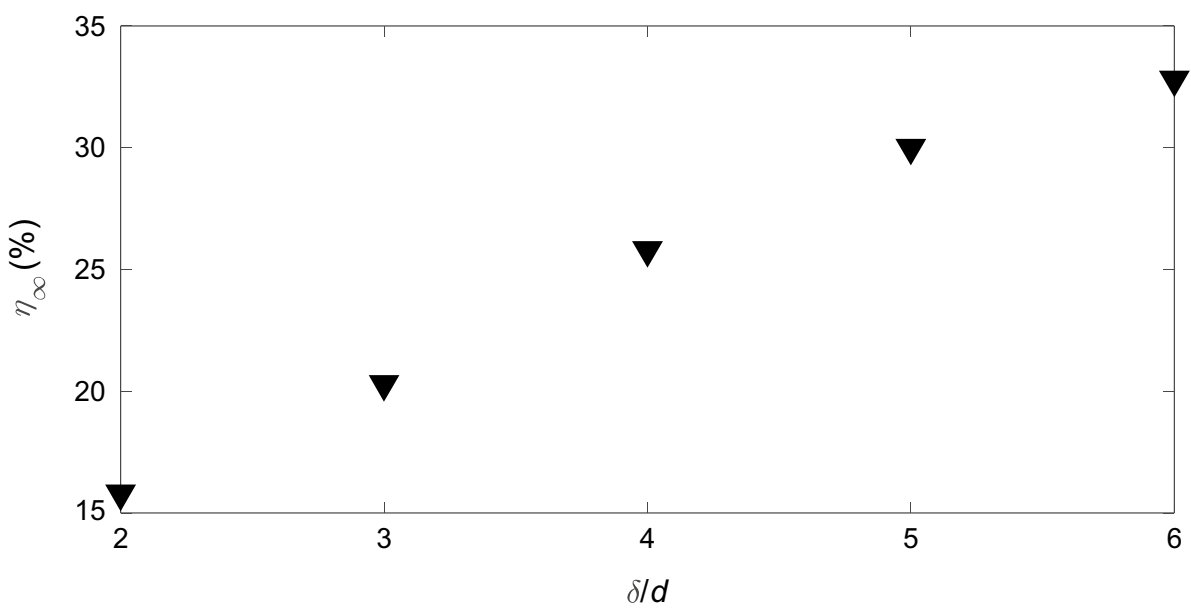



on the three packing fraction reduction mechanisms, $v_{w}=100 \mathrm{~mm} / \mathrm{s}$

Fig. 12 Effect of gap thickness on the ratio of packing fraction between the front and back sublayers, $v_{w}=100 \mathrm{~mm} / \mathrm{s}$
Fig. 11 Effect of gap thickness
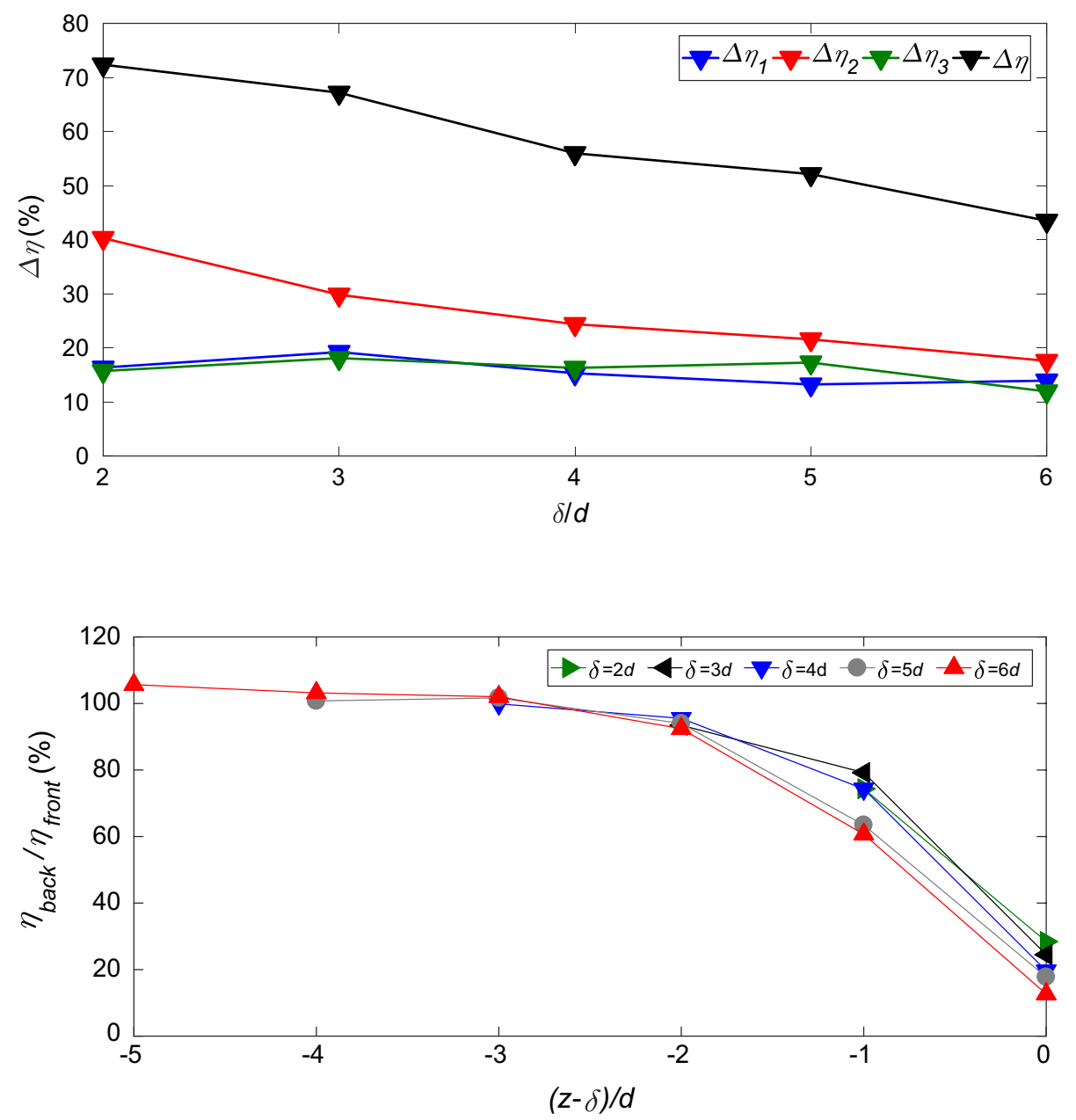

Fig. 13 Effect of gap thickness on the particle $x$-velocity profile inside the shear band in front of the spreader, $v_{w}=100 \mathrm{~mm} / \mathrm{s}$

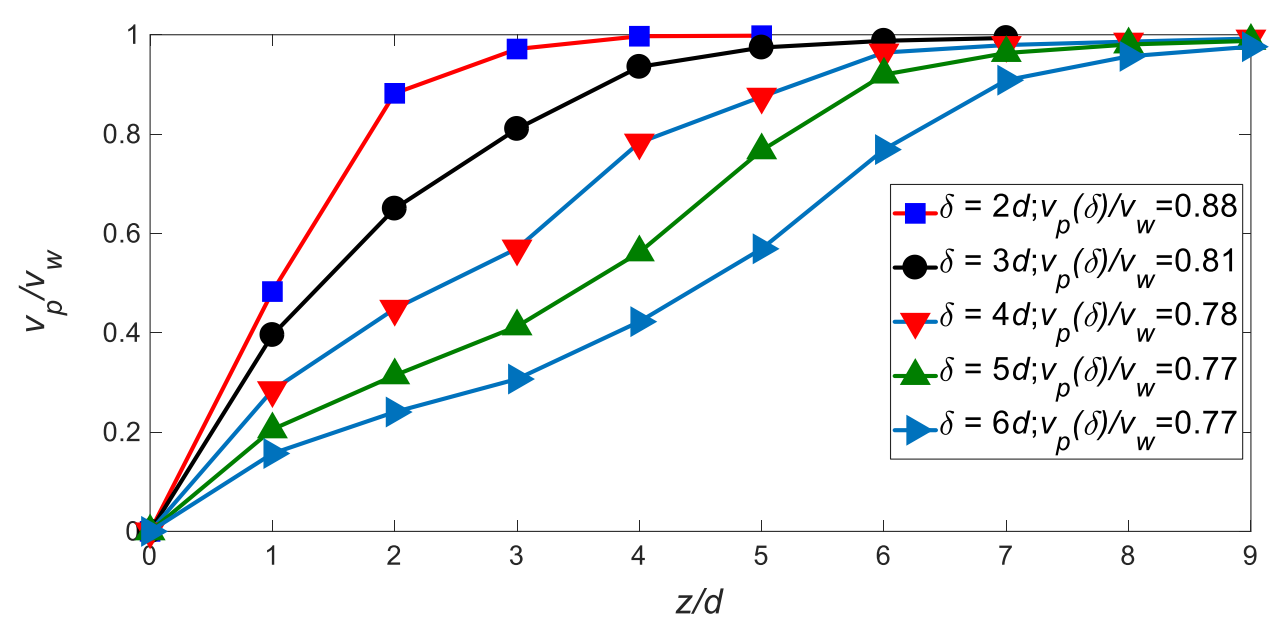

is nearly constant between 16 and $18 \%$ for gap thicknesses between 2 and 5 particle diameters. It drops slightly for 6 particle diameters to reach $12 \%$. The mean velocity in the shear layer decreases as the gap height increases, see Fig. 13, consequently a smaller reduction in packing fraction would be anticipated with large gaps.
In order to further assess the second mechanism, Fig. 12 shows the effect of gap thickness on the ratio of packing fraction between the front and back sub-layers. The sublayers numbers are reversed with a reference point at the top of the gap so that horizontal axis represents $(z-\delta) / d$ ( 0 is the top sub-layer index). Interestingly, very similar 
profiles are observed with zone where the reduction in packing fraction is seen being independent of gap height. For all gap thicknesses, most of the reduction in packing fraction occurs within the top two sub-layers with the packing fraction at the back reaching around 95\% of that of the front in the -2 sub-layer. Surprisingly, the dilation in the top sub-layers is higher for larger gap thicknesses. This indicates that the increase in the final packing fraction of the deposited layer with the gap thickness (Fig. 10) is due to the increase in the number of the bottom sub-layers in which the packing fraction is remaining unchanged as the particles exit the gap. The vertical flux of powder must maintain the packing fraction roughly constant in all but the top two layers. For a gap thickness of 2 particle diameter, the dilated zone occupies the whole gap, which means that there is no unaffected sub-layers at the bottom of the gap as noticed for larger gaps.

Figure 13 shows the effect of gap thickness on the particle $x$-velocity profile inside the shear band in front of the spreader. As expected, the average shear rate across the gap, defined as the ratio between the variation of the velocity across the gap and the gap thickness, decreases by increasing the gap thickness. It is also clear that the wall slip velocity increases with decreasing the gap thickness. This is consistent with the observations of Siavoshi et al. [55] in the study of confined shear of granular particles in thin gaps. Above the gap, the average shear rate decreases significantly and there is an asymptotic approach to the value of the spreader velocity. This asymptotic approach of the particle velocity to the spreader velocity occurs over a smaller length scale for the smallest gap. An $S$-shaped velocity profile [56] starts to emerge and becomes more pronounced with larger gap thickness. The increase in the wall slip at lower gap heights is important as it leads to an increase in the mean velocity in the front region. This causes a decrease in the particle velocity relative to the blade which, via conservation of mass, Eq. (1), is directly correlated to the final layer packing fraction.
The results presented in this section have shown that the final layer packing fraction increases with the gap thickness. It has been shown that the contribution from the second mechanism, powder flow through the gap, is dominant; though its relative contribution compared to the two other mechanisms decreases with the gap thickness. As the gap thickness increases, the number of unaffected bottom layers increases and the reduction of the packing fraction at the back of the spreader is localised in the top two sub-layers of the gap. The influence of the mean horizontal velocity in the gap has also been highlighted.

\subsubsection{Spreader velocity}

The spreader velocity is an important process parameter that might affect the powder spreading process. It is desirable to increase the spreader velocity so that the spreading process contributes less to the overall build time, but it was reported in the literature that a higher spreader velocity reduces the quality of the deposited layer [13,14]. It is thus important to understand the effect of the spreader velocity on the mechanics of the spreading and the final layer thickness to identify the best compromise between the manufacturing time and the layer quality. Varying the spreader velocity varies the shear rate of the particles inside the gap. Here, five different spreader velocities are tested: $10,30,50,80$ and $100 \mathrm{~mm} / \mathrm{s}$. For all results presented in this section, the layer thickness is kept constant at 4 particle diameters $(0.2 \mathrm{~mm})$.

Figure 14 shows the effect of the spreader velocity on the final layer packing fraction. The packing fraction decreases with spreader velocity in an almost linear fashion. Figure 15 shows the effect of the spreader velocity on the three packing fraction reduction mechanisms, all of which show a larger reduction with increasing spreader velocity. For the first mechanism, shear-induced dilation during the initiation of powder motion by the spreader, the deposited layer packing fraction reduction increases linearly with the spreader velocity, though its slope is small with $8 \%$ dilation for
Fig. 14 Effect of spreader velocity on the final layer packing fraction, $\delta=4 d$

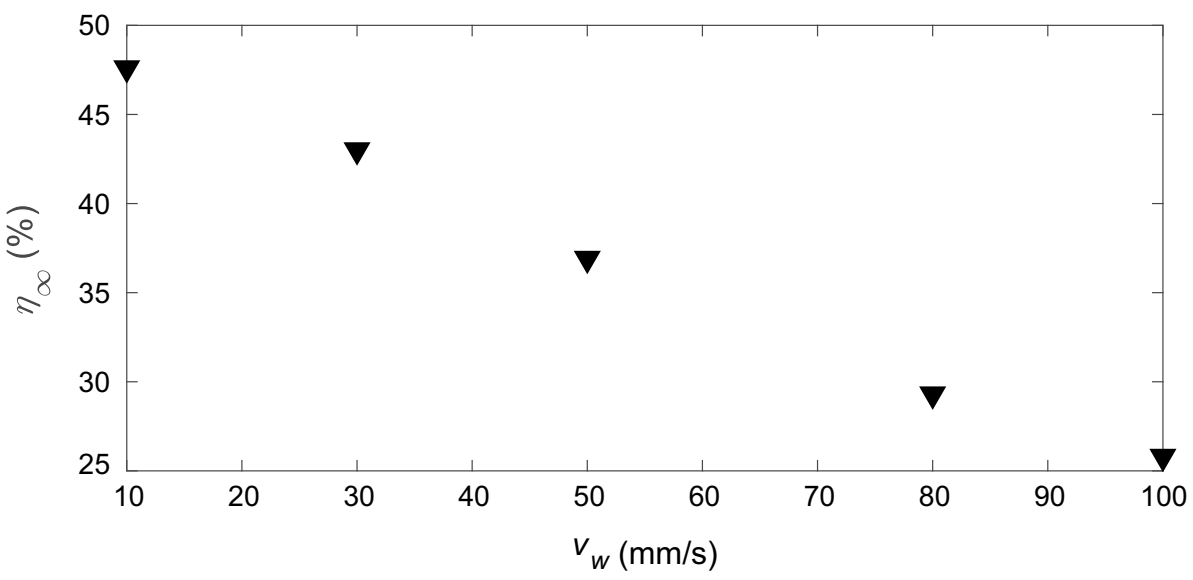


Fig. 15 Effect of the spreader velocity on the three packing fraction reduction mechanisms, $\delta=4 d$

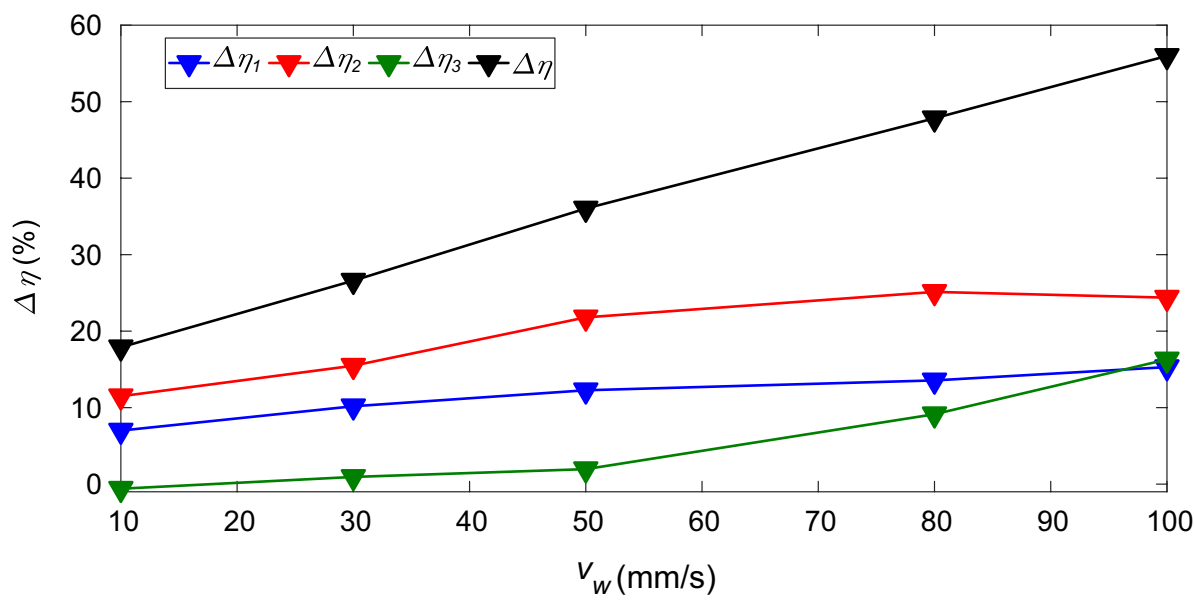

the minimum spreader velocity of $10 \mathrm{~mm} / \mathrm{s}$ and $15 \%$ for the maximum spreader velocity of $100 \mathrm{~mm} / \mathrm{s}$. The second mechanism, dilation and rearrangement when the powder moves through the gap, is the most significant mechanism for all spreader velocities. The shear induced dilation associated with these two mechanisms increases with the spreader velocity (shear rate); in agreement with the trend reported in the literature of plane shear flow [57]. At very low spreader velocities, the packing fraction reduction due to the velocity change (third mechanism) is almost zero for a spreader velocity of 10,30 and $50 \mathrm{~mm} / \mathrm{s}$, indicating low particle velocities at the back of the spreader in these cases. As the spreader velocity increases, the particle inertia increases and the contribution from the third mechanism increases. Interestingly, the overall reduction in packing fraction exhibits almost linear relation with the spreader velocity.

Figure 16 shows the effect of spreader velocity on the ratio of packing fraction between the front and back sub-layers (second mechanism). For the lowest velocities, $10 \mathrm{~mm} / \mathrm{s}$ and $30 \mathrm{~mm} / \mathrm{s}$, the reduction in the packing fraction is localised in the top sub-layer, and the packing fraction of the bottom sub-layers can be slightly higher at the back compared to that at the front indicating that some reorganisation and improve packing takes place in this sub-layer, and is perhaps indicative of lower shear induced dilation decrease in these low velocity cases. Overall, the depth of dilation region increases with velocity, and begins to extend to the second sub-layer. The increase in dilation of the third and fourth sub-layers is not always a monotonic increase with velocity indicating several competing effects.

Figure 17 shows the effect of the spreader velocity on the particle $x$-velocity profile inside the shear band in front of the spreader. It is clear that the normalised slip velocity at the wall increases with the spreader velocity, and that the mean normalised velocity also increases, a trend noticed also when the gap thickness was decreased (Fig. 7). The jump in the normalised velocity at the edge of the gap thickness (from layer 3 to 4 ) is highest with the lowest spreader velocity and decreases with increasing the spreader velocity. Above the spreader, the profiles taper and converge towards the spreader velocity reaching it at 9 particle diameters. The decrease in the normalised slip velocity at the wall results in a more pronounced $S$-shaped velocity profile. The emergence of this $S$-shaped velocity profile is due to the lower
Fig. 16 Effect of spreader velocity on the ratio of packing fraction between the front and back sub-layers, $\delta=4 d$

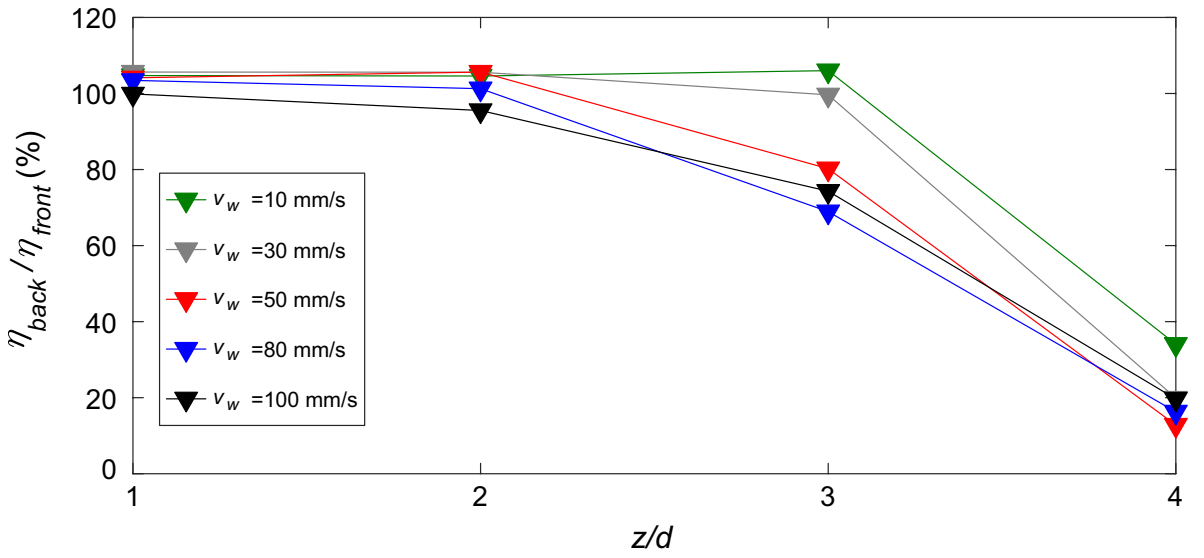


Fig. 17 Effect of the spreader velocity on the particle $x$-velocity profile inside the shear band in front of the spreader, $\delta=4 d$

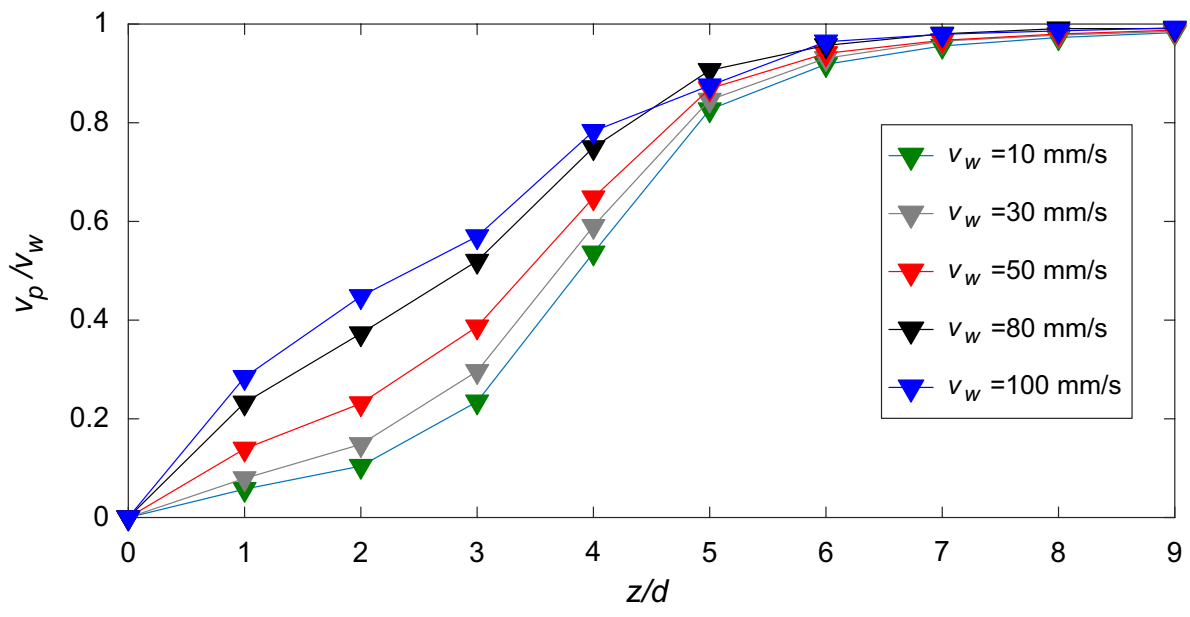

velocity gradient at the vicinity of the build surface. This $S$-shaped velocity profile was reported in double plane shear [56] and the decrease in the slip velocity as the shear velocity decreases following a similar trend of plane simple shear was reported by Wang et al. [58].

The results presented in this section have shown that the final layer packing fraction decreases with the spreader velocity in almost a linear fashion. The increase in the spreader velocity increases the shear rate. Thus, the shear induced dilation becomes more pronounced for higher spreader velocities. The reduction caused the third mechanism, due to particle inertia, to increase significantly with the spreader velocity as it was almost zero for relatively low spreader velocities of 10,30 and $50 \mathrm{~mm} / \mathrm{s}$. It reaches $15 \%$ for the highest velocity $100 \mathrm{~mm} / \mathrm{s}$.

\section{Summary and conclusions}

In this paper, DEM simulations were used to study the mechanics of powder spreading in an idealised, blade based, AM system. A vertical spreader blade pushed the powder along a horizontal surface, creating a shear band whose height in front of the spreader was 9 particle diameters. Particles move through the gap to the back of the spreader and subsequently stop to create a thin powder layer. Three mechanisms which lead to an inevitable reduction of the deposited layer packing fraction compared to that of the initial heap were identified. The first mechanism is the initial shear-induced dilation during the initiation of powder motion by the spreader. The second mechanism is due to the dilation and rearrangement of the powder when it moves through the gap. The third mechanism is due to the mass conservation as the particles decelerate and become stationary in the deposited powder layer. It was shown that varying either the gap thickness or the spreader velocity can control the final packing fraction via suppressing or promoting one or more of these mechanisms. The final layer packing fraction increases with the gap thickness, and decreases with the spreader velocity. The velocity profile that is generated in the gap is directly responsible for the final layer packing fraction and the mean velocity in this region dictates the final layer packing fraction. The analysis presented in this idealised study can help to create a framework for rigorously studying and optimising the process of powder spreading in AM. It could be used to understand the role of particle properties, such as size distribution, morphology and cohesivity, and their interaction with process parameters. One key challenge in the application to more realistic systems is the description of the recently melted layer onto which the particles are being spread, in particular the morphology i.e. roughness, friction, cohesion at the operating temperature of the AM system.

Acknowledgements The authors are grateful to the staff of 'GKN Aerospace AM Filton Technology Centre' for introducing their AM machines; in specific we thank Dr Alphons Antonysamy and Dr Mahesh Mani for the valuable discussions. Funding for this work was provided by the ATI (Aerospace Technology Institute), through the TiPOW (Titanium Powder for Net-shape Component Manufacture) project, and by the EPSRC through the MAPP (Manufacture Using Advanced Powder Processes) Future Manufacturing Hub (Grant Ref. $\mathrm{EP} / \mathrm{P} 006566 / 1)$

\section{Compliance with ethical standards}

Conflict of interest The authors declare that they have no conflict of interest.

Open Access This article is distributed under the terms of the Creative Commons Attribution 4.0 International License (http://creativeco mmons.org/licenses/by/4.0/), which permits unrestricted use, distribution, and reproduction in any medium, provided you give appropriate credit to the original author(s) and the source, provide a link to the Creative Commons license, and indicate if changes were made. 


\section{References}

1. ASTM, ASTM F2792-12a: Standard Terminology for Additive Manufacturing Technologies, ASTM International, West Conshohocken, PA (2012)

2. Markillie, P.: A third industrial revolution. The Economist 21 (2012). https://www.economist.com/node/21552901

3. Vandenbroucke, B., Kruth, J.P.: Selective laser melting of biocompatible metals for rapid manufacturing of medical parts. Rapid Prototyp. J. 13, 196-203 (2007)

4. Herzog, D., Seyda, V., Wycisk, E., Emmelmann, C.: Additive manufacturing of metals. Acta Mater. 117, 371-392 (2016)

5. Antonysamy, A.A.: Microstructure, texture and mechanical property evolution during additive manufacturing of Ti6Al4V alloy for aerospace applications, Ph.D. thesis, University of Manchester (2012)

6. Yablokova, G., Speirs, M., Van Humbeeck, J., Kruth, J.P., Schrooten, J., Cloots, R., Boschini, F., Lumay, G., Luyten, J.: Rheological behavior of $\beta$-Ti and NiTi powders produced by atomization for SLM production of open porous orthopedic implants. Powder Technol. 283, 199-209 (2015)

7. Gong, X., Anderson, T., Chou, K.: Review on powder-based electron beam additive manufacturing technology. Manuf. Rev. 1, 2 (2014)

8. Dawes, J., Bowerman, R., Trepleton, R.: Introduction to the additive manufacturing powder metallurgy supply chain. Johnson Matthey Technol. Rev. 59, 243-256 (2015)

9. Spierings, A.B., Voegtlin, M., Bauer, T., Wegener, K.: Powder flowability characterisation methodology for powder-bed-based metal additive manufacturing. Prog. Addit. Manuf. 1, 9-20 (2016)

10. Spierings, A.B., Herres, N., Levy, G.: Influence of the particle size distribution on surface quality and mechanical properties in AM steel parts. Rapid Prototyp. J. 17, 195-202 (2011)

11. Sun, C., Tian, X., Wang, L., Liu, Y., Wirth, C.M., Günster, J., Li, D., Jin, Z.: Effect of particle size gradation on the performance of glass-ceramic 3D printing process. Ceram. Int. 43, 578-584 (2017)

12. Spierings, A., Levy, G.: Comparison of density of stainless steel $316 \mathrm{~L}$ parts produced with selective laser melting using different powder grades. In: Proceedings of the Annual International Solid Freeform Fabrication Symposium, Austin, TX, pp. 342-353 (2009)

13. Parteli, E.J.R., Pöschel, T.: Particle-based simulation of powder application in additive manufacturing. Powder Technol. 288, 96-102 (2016)

14. Haeri, S., Wang, Y., Ghita, O., Sun, J.: Discrete element simulation and experimental study of powder spreading process in additive manufacturing. Powder Technol. 306, 45-54 (2017)

15. Xiang, Z., Yin, M., Deng, Z., Mei, X., Yin, G.: Simulation of forming process of powder bed for additive manufacturing. $\mathrm{J}$. Manuf. Sci. Eng. 138, 081002 (2016)

16. Haeri, S.: Optimisation of blade type spreaders for powder bed preparation in additive manufacturing using DEM simulations. Powder Technol. 321, 94-104 (2017)

17. Cundall, P.A., Strack, O.D.L.: A discrete numerical model for granular assemblies. Géotechnique 29, 47-65 (1979)

18. Tsuji, Y., Tanaka, T., Ishida, T.: Lagrangian numerical simulation of plug flow of cohesionless particles in a horizontal pipe. Powder Technol. 71, 239-250 (1992)

19. EDEM 2017 User Guide. DEM Solutions Ltd., Edinburgh, Scotland, UK. Copyright (C) (2016)

20. Thakur, S.C., Morrissey, J.P., Sun, J., Chen, J.F., Ooi, J.Y.: Micromechanical analysis of cohesive granular materials using the discrete element method with an adhesive elasto-plastic contact model. Granular Matter 16, 383-400 (2014)
21. Hassanpour, A., Tan, H., Bayly, A., Gopalkrishnan, P., Ng, B., Ghadiri, M.: Analysis of particle motion in a paddle mixer using discrete element method (DEM). Powder Technol. 206, 189-194 (2011)

22. Arratia, P.E., Duong, N.-H., Muzzio, F.J., Godbole, P., Reynolds, S.: A study of the mixing and segregation mechanisms in the Bohle Tote blender via DEM simulations. Powder Technol. 164, 50-57 (2006)

23. Yuu, S., Abe, T., Saitoh, T., Umekage, T.: Three-dimensional numerical simulation of the motion of particles discharging from a rectangular hopper using distinct element method and comparison with experimental data (effects of time steps and material properties). Adv. Powder Technol. 6, 259-269 (1995)

24. Hare, C., Zafar, U., Ghadiri, M., Freeman, T., Clayton, J., Murtagh, M.J.: Analysis of the dynamics of the FT4 powder rheometer. Powder Technol. 285, 123-127 (2015)

25. Rackl, M., Hanley, K.J.: A methodical calibration procedure for discrete element models. Powder Technol. 307, 73-83 (2017)

26. Combarros, M., Feise, H.J., Zetzener, H., Kwade, A.: Segregation of particulate solids: experiments and DEM simulations. Particuology 12, 25-32 (2014)

27. Grima, A.P., Wypych, P.W.: Development and validation of calibration methods for discrete element modelling. Granul. Matter 13, 127-132 (2011)

28. Derakhshani, S.M., Schott, D.L., Lodewijks, G.: Micro-macro properties of quartz sand: experimental investigation and DEM simulation. Powder Technol. 269, 127-138 (2015)

29. Coetzee, C.J.: Calibration of the discrete element method and the effect of particle shape. Powder Technol. 297, 50-70 (2016)

30. Paulick, M., Morgeneyer, M., Kwade, A.: Review on the influence of elastic particle properties on DEM simulation results. Powder Technol. 283, 66-76 (2015)

31. Frankowski, P., Morgeneyer, M.: Calibration and validation of DEM rolling and sliding friction coefficients in angle of repose and shear measurements. AIP Conf. Proc. 1542, 851-854 (2013)

32. Cleary, P.W.: DEM prediction of industrial and geophysical particle flows. Particuology 8, 106-118 (2010)

33. Lommen, S., Schott, D., Lodewijks, G.: DEM speedup: stiffness effects on behavior of bulk material. Particuology 12, 107-112 (2014)

34. Karapatis, P.: A sub-process approach of selective laser sintering, Ph.D. thesis, EPFL (2002)

35. MiDi, G.: On dense granular flows. Eur. Phys. J. E 14, 341-365 (2004)

36. Mueth, D.M., Debregeas, G.F., Karczmar, G.S., Eng, P.J., Nagel, S.R., Jaeger, H.M.: Signatures of granular microstructure in dense shear flows. Nature 406, 385-389 (2000)

37. Murdoch, N., Rozitis, B., Green, S.F., de Lophem, T.-L., Michel, P., Losert, W.: Granular shear flow in varying gravitational environments. Granul. Matter 15, 129-137 (2013)

38. Mueth, D.M.: Measurements of particle dynamics in slow, dense granular Couette flow. Phys. Rev. E 67, 011304 (2003)

39. Reynolds, O.: LVII. On the dilatancy of media composed of rigid particles in contact. With experimental illustrations. Philos. Mag. Ser. 20, 469-481 (1885)

40. Alexander, A.W., Chaudhuri, B., Faqih, A., Muzzio, F.J., Davies, C., Tomassone, M.S.: Avalanching flow of cohesive powders. Powder Technol. 164, 13-21 (2006)

41. Boonkanokwong, V., Remy, B., Khinast, J.G., Glasser, B.J.: The effect of the number of impeller blades on granular flow in a bladed mixer. Powder Technol. 302, 333-349 (2016)

42. Faqih, A., Chaudhuri, B., Muzzio, F.J., Tomassone, M.S., Alexander, A., Hammond, S.: Flow-induced dilation of cohesive granular materials. AIChE J. 52, 4124-4132 (2006)

43. Fullard, L.A., Davies, C.E., Lube, G., Neather, A.C., Breard, E.C.P., Shepherd, B.J.: The transient dynamics of dilation waves 
in granular phase transitions during silo discharge. Granul. Matter 19, 6 (2016)

44. Hurley, R.C., Andrade, J.E.: Friction in inertial granular flows: competition between dilation and grain-scale dissipation rates. Granul. Matter 17, 287-295 (2015)

45. Oda, M., Kazama, H.: Microstructure of shear bands and its relation to the mechanisms of dilatancy and failure of dense granular soils. Géotechnique 48, 465-481 (1998)

46. Fickie, K.E., Mehrabi, R., Jackson, R.: Density variations in a granular material flowing from a wedge-shaped hopper. AIChE J. 35, 853-855 (1989)

47. Le Pennec, T., Maloy, K.J., Flekkoy, E.G., Messager, J.C., Ammi, M.: Silo hiccups: dynamic effects of dilatancy in granular flow. Phys. Fluids 10, 3072 (1998)

48. Drescher, A., Cousens, T.W., Bransby, P.L.: Kinematics of the mass flow of granular material through a plane hopper. Géotechnique 28, 27-42 (1978)

49. Lee, J., Cowin, S.C., Templeton, J.S.: An experimental study of the kinematics of flow through hoppers. Trans. Soc. Rheol. 18, 247 (1974)

50. Michalowski, R.L.: Flow of granular material through a plane hopper. Powder Technol. 39, 29-40 (1984)

51. Weir, G.J.: A mathematical model for dilating, non-cohesive granular flows in steep-walled hoppers. Chem. Eng. Sci. 59, 149-161 (2004)

52. Zhou, Y., Lagrée, P.-Y., Popinet, S., Ruyer, P., Aussillous, P.: Experiments on, and discrete and continuum simulations of, the discharge of granular media from silos with a lateral orifice. J. Fluid Mech. 829, 459-485 (2017)

53. Janda, A., Harich, R., Zuriguel, I., Maza, D., Cixous, P., Garcimartín, A.: Flow-rate fluctuations in the outpouring of grains from a two-dimensional silo. Phys. Rev. E 79, 031302 (2009)

54. Prakash, J.R., Ravi, J., Rao, K.K.: Steady compressible flow of cohesionless granular materials through a wedge-shaped bunker. J. Fluid Mech. 225, 21-80 (1991)

55. Siavoshi, S., Orpe, A.V., Kudrolli, A.: Friction of a slider on a granular layer: nonmonotonic thickness dependence and effect of boundary conditions. Phys. Rev. E 73, 010301 (2006)

56. Miller, T., Rognon, P., Metzger, B., Einav, I.: Eddy viscosity in dense granular flows. Phys. Rev. Lett. 111, 058002 (2013)

57. da Cruz, F., Emam, S., Prochnow, M., Roux, J.-N., Chevoir, F.: Rheophysics of dense granular materials: discrete simulation of plane shear flows. Phys. Rev. E 72, 021309 (2005)

58. Wang, X., Zhu, H.P., Yu, A.B.: Flow properties of particles in a model annular shear cell. Phys. Fluids 24, 053301 (2012)

Publisher's Note Springer Nature remains neutral with regard to jurisdictional claims in published maps and institutional affiliations. 